\title{
1 Two helices control the dynamic crosstalk between the catalytic domains of LRRK2
}

3 Jui-Hung Weng ${ }^{1^{*}}$, Phillip C. Aoto ${ }^{1^{*}}$, Robin Lorenz ${ }^{2}$, Jian $\mathrm{Wu}^{1}$, Sven H. Schmidt², Jascha T. Manschwetus ${ }^{2}$, Pallavi

4 Kaila-Sharma ${ }^{1}$, Sebastian Mathea ${ }^{3}$, Stefan Knapp ${ }^{3}$, Friedrich W. Herberg ${ }^{2}$, Susan S. Taylor ${ }^{1,4, a}$

$5 \quad{ }^{1}$ Department of Pharmacology, University of California, San Diego, USA

$6 \quad 2$ Department of Biochemistry, University of Kassel, Germany

$7 \quad{ }^{3}$ Institute for Pharmaceutical Chemistry, Goethe University Frankfurt, Germany

${ }^{4}$ Department of Chemistry and Biochemistry, University of California, San Diego, USA

$12{ }^{*}$ Authors contributed equally

13 a corresponding author

14 Email: staylor@ucsd.edu 


\section{Abstract}

17 The two major molecular switches in biology, kinases and GTPases, are both contained in the

18 Parkinson's Disease-related Leucine-rich repeat kinase 2 (LRRK2). Using hydrogen-deuterium

19 exchange mass spectrometry (HDX-MS) and Molecular Dynamics (MD) simulations, we

20 generated a comprehensive dynamic allosteric portrait of the C-terminal domains of LRRK2

21 (LRRK2 $\left.2_{\text {RCKW }}\right)$. We identified two helices that shield the kinase domain and regulate LRRK2

22 conformation and function. One docking helix in COR-B (Dk-Helix) tethers the COR-B domain to

23 the $\alpha \mathrm{C}$ helix of the kinase domain and faces its Activation Loop, while the C-terminal helix (Ct-

24 Helix) extends from the WD40 domain and interacts with both kinase lobes. The Ct-Helix and

25 the N-terminus of the Dk-Helix create a "cap" that regulates the N-Lobe of the kinase domain.

26 Our analyses reveal allosteric sites for pharmacological intervention and confirm the kinase

27 domain as the central hub for conformational control. 


\section{Introduction}

Parkinson's Disease (PD), a major neurodegenerative disorder, is characterized by

31 chronic and progressive loss of dopaminergic neurons. Mutations in the PARK 8 gene which

32 codes for the Leucine-Rich Repeat Kinase 2 (LRRK2) are the most common cause for genetically driven PD[1]. LRRK2 is a large multi-domain protein that contains an armadillo repeat motif

34 (ARM), ankyrin repeat (ANK), leucine-rich repeat (LRR), ras-of-complex (ROC) GTPase, Cterminal of ROC (COR), protein kinase, and WD40 domains[2]. While crosstalk between kinases and GTPases, the two most important molecular switches in biology, are well-known features in

37 cellular signaling, LRRK2 is one of the few proteins that contains both catalytic domains in the same polypeptide chain[3]. GTP binding to the ROC domain is thought to regulate kinase activity as well as stability and localization[4,5]. Most of the well-known familial mutations are clustered within the ROC, COR and kinase domains; N1473H and R1441C/G/H in the GTPase

41 domain and Y1699C in COR-B lie at the interface between the ROC and COR domains, while

42 G2019S and I2020T are in the highly conserved DFG $\psi$ motif within the kinase domain[6,7]. This information collectively suggests that there is considerable crosstalk between the two catalytic domains of LRRK2, but can we capture this crosstalk? molecular switch. Its conformation regulates more than just kinase activity and plays a crucial

47 role in the intrinsic regulatory processes that mediate subcellular location and activation of LRRK2[8]. Recent breakthroughs in obtaining structure information, including the in situ cryo 
the high resolution cryo electron microscopy (cryo-EM) structure of the catalytic C-terminal

51 domains (LRRK2 $\left.2_{\mathrm{RCKW}}\right)$, have provided invaluable structural templates that enabled us to achieve

52 a mechanistic understanding of LRRK2[9,10]. Most recently the cryo-EM structure of full length

53 LRRK2 was also solved at high resolution[11].

Here, we combined hydrogen-deuterium exchange mass spectrometry (HDX-MS) and

Gaussian Accelerated Molecular Dynamics (GaMD) simulations to gain insight into the dynamic

56 features of LRRK2 $2_{R C K W}$, a construct that includes both the kinase and GTPase domains. To build

57 a comprehensive allosteric and dynamic portrait of $L R R K 2_{R C K W}$, we first mapped our HDX-MS

data onto the LRRK2 $2_{\mathrm{RCKW}}$ cryo-EM structure which gave us a portrait of the solvent accessibility

59 of each peptide. We also assessed the effect of the type I kinase inhibitor MLi-2 and finally used

60

61

62

63

64

65

66

67

68

69

70
GaMD simulations to monitor the dynamics of LRRK2 $2_{R C K W}$.

The intrinsic dynamic features of LRRK2 $2_{R C K W}$ revealed by HDX-MS and GaMD simulations show how the kinase domain is allosterically regulated by its flanking domains. These two techniques allow us to explore the molecular features of domain:domain interfaces and loop dynamics. In this way we identified two distinct motifs that control the kinase domain. These two motifs, the COR-B docking helix (referred to as the Dk-Helix) and the C-terminal helix (CtHelix), both impact the overall breathing dynamics of LRRK $2_{R C K W}$. In addition, we showed how the Activation Segment (AS) of the kinase domain faces the ROC:COR-B interface. This interface is unleashed by several PD mutations that cluster in the kinase domain and at the interface between COR-B and the ROC domain. The AS is disordered in the LRRK $2_{\text {RCKW }}$ cryo-EM structure, and GaMD simulations allowed us to explore this space. In this inactive conformation, the Dk- 
71 Helix is stably anchored onto the $\alpha \mathrm{C}$ helix in the $\mathrm{N}$-lobe of the kinase domain, which locks the

$72 \alpha C$ helix into an inactive conformation.

\section{Results}

\section{Global dynamic portrait of LRRK2 $2_{\text {RCKW }}$ is revealed by HDX-MS and GaMD simulations}

To identify the solvent exposed regions of LRRK2 $2_{R C K W}$, we mapped the HDX-MS data onto the cryo-EM structure of LRRK2 ${ }_{\mathrm{RCKW}}[10]$ (Fig 1a). Our overall HDX-MS coverage of LRRK2 $2_{\text {RCKW, }}$ which was $>98 \%$ (S1 Fig), shows the relative fractional deuterium uptake of each peptide. We previously mapped the HDX-MS profile onto a model of the kinase domain[12] while here we mapped the HDX-MS exchange pattern onto the entire LRRK2 $2_{R C K W}$ Cryo-EM structure. This allows us to capture the crosstalk between and within the C-terminal domains. As shown in S2a Fig., the C-lobe of the kinase domain around the Activation Loop (A-Loop), a part of the surface region of the ROC domain, and parts the COR-B domain show the highest deuterium uptake. These regions are either highly flexible or likely to be unfolded, and some

85 are not resolved in the static cryo-EM structure[10]. In contrast, the core of the ROC domain and the core of the COR-B domain show low deuterium uptake suggesting that they are wellfolded and form rigid domains S2b Fig. The WD40 domain, except for several loops, also has less deuterium uptake, which indicates that its core is less dynamic in solution.

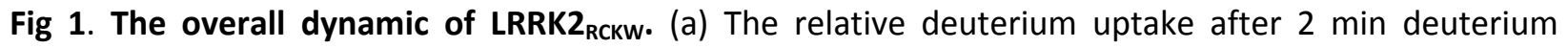


91 information. The surface of the kinase domain is shown in gray. The highly protected Dk-Helix and the

92 Ct-Helix located at the back of the kinase domain are labelled. (b) Snapshot of LRRK2 $2_{R C K W}$ in the MD

93 simulation. The surface of each domain is shown in different colors. Left represents the compact

94 architecture of $\mathrm{LRRK} 2_{\mathrm{RCKW}}$. The middle is the structure from cryo-EM. The right represents one of the

95 extended states of LRRK2 $2_{\text {RCKW }}$

There are also regions on the surface of each domain that are highly protected from

97 solvent suggesting that these are domain-domain interfaces. The N-lobe of the kinase domain,

98 for example, is relatively well shielded from solvent in contrast to the highly exposed C-lobe S2b

(S2b Fig). The two helices of the COR-A domain at the interface contacting the ROC domain are

mostly shielded, indicating that the interaction between COR-A and the ROC domain is stable

101

102

103

104

105

106

107

108

109

and persistent. One of the ROC domain helices and the adjacent loops of the COR-B domain all have low deuterium uptake, indicating that the ROC:COR-B interface is also shielded from solvent in this inactive conformation. Parts of the kinase domain N-lobe with low deuterium uptake are mostly shielded by the COR-B-Kinase linker and the COR-B domain. Another interface that is well shielded from solvent lies between the kinase and WD40 domain. The beginning of the WD40 domain, the $\mathrm{N}$-terminal end of the $\alpha \mathrm{E}$ helix in the kinase domain and the N-terminus of the C-terminal helix, which extends from the WD40 domain, all have very low deuterium uptake suggesting that the WD40 domain interacts persistently with the C-lobe of the kinase in solution (S2b Fig). This also explains why a stable and active isolated kinase domain of LRRK2 has not been expressed yet.

To investigate the dynamic features of $L R R K 2_{R C K W}$ and the interactions within its domains, we performed GaMD simulations to recapitulate the behavior of $L R R K 2_{R C K W}$ in solution. 
113 To capture a more accurate representative model of LRRK2 $2_{R C K W}$ breathing dynamics, we applied

114 enhanced sampling to broadly sample the conformational changes that take place during the

115 simulations. Both extended and compact conformations of LRRK2 $2_{R C K W}$ are captured by the

116 simulations (Fig. 1b), and the kinase domain is at the center of the breathing dynamics of

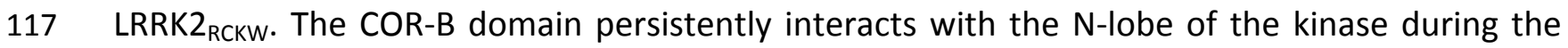

118 simulation while the WD40 domain interacts stably with the C-lobe of the kinase domain. The

119 COR-A and ROC domains move as a single rigid body and fluctuate between far and near states

120 relative to the $\mathrm{C}$-lobe of the kinase domain. When the kinase domain is in a closed

121 conformation, the ROC domain and the COR-A domain are brought closer to the C-lobe of the

122 kinase domain (Fig. 1b, left) while in an open conformation (Fig 1b, right), the COR-A domain

123 and ROC domain move further away from the C-lobe of the kinase domain thereby creating a

124 more extended conformation. The dynamic features that bring the ROC domain and the C-lobe

125 of the kinase domain into close proximity correlate with the crosstalk between the kinase and

126 GTPase domains. As described below, our GaMD simulations also revealed many interactions

127 that are potentially involved in allosteric crosstalk within LRRK2 as well as how these

128 interactions might be influenced by PD mutations.

129 Domain interfaces with the kinase domain.

130 To better understand how the kinase domain is shielded from solvent, we focused on two

131 dominant helices that embrace the $\mathrm{N}$ - and C-lobes of the kinase domain (S3 Fig.). One lies in the

132 COR-B domain (residues 1771-1791) and is buttressed up against the $\alpha \mathrm{C}$ helix in the $\mathrm{N}$-lobe

133 while the other helix (residues 2500-2527) lies at the C-terminus and is anchored mainly to the

134 C-lobe of the kinase domain. We refer to these as the COR-B docking helix (or Dk-Helix) and the 
135 C-terminal helix (or Ct-Helix), respectively. In contrast to the well-shielded N-lobe, much of the

136 C-lobe is disordered in the cryo-EM LRRK2 $2_{R C K W}$ structure, which represents an inactive

137 conformation. In the kinase domain, we focus on the extended Activation Segment (AS), which

138 is typically well-ordered in active kinases and poised to interact with substrates and

139 inhibitors[13]. In the LRRK2 $2_{\text {RCKW }}$ structure, the disordered A-Loop at the beginning of the AS

140 faces the Dk-Helix, while the region at the end of the AS faces ROC and COR-A (S3. Fig.).

141 Capturing crosstalk between the kinase and COR-B domains.

142 The COR-B domain plays a critical role in coordinating the communication that takes place

143 between the kinase domain and the ROC domain. Based on our HDX-MS results (Fig. 2), the

144 interface between the N-lobe of the kinase domain and the COR-B domain is mostly shielded

145 from solvent. This surface is dominated by a long amphipathic helix, the Dk-Helix, and the $\alpha \mathrm{C}$

146 helix of the kinase domain (Fig. 2). The Dk-Helix can be divided into three segments based on

147 HDX-MS. While the middle region (residues 1777-1788) shows almost no uptake, the N-

148 terminus shows a slow deuterium exchange. In contrast, the C-terminus of the Dk-Helix is more

149 exposed to solvent but also close to both the disordered A-Loop of the kinase domain and to

150 the ROC domain. It has approximately $50 \%$ deuterium uptake at $2 \mathrm{~min}$, which is reduced to $35 \%$

151 when the type I LRRK2 kinase inhibitor MLi-2 is bound. The N-terminus of the $\alpha \mathrm{C}$ helix in the

152 kinase domain that binds to the Dk-Helix shows an unusual uptake pattern. The uptake

153 increases at a linear rate signifying a slow exchange without reaching a plateau within 2 min,

154 while binding of MLi-2 significantly protects against uptake. Together, HDX-MS shows that the

155 COR-B:kinase domain interface is mostly shielded from solvent and that the Dk-Helix, in

156 particular, is stably anchored to the $\mathrm{N}$-lobe of the kinase while the $\mathrm{N}$-terminus of the Dk-Helix is 
157 dynamic and communicates with both the kinase and ROC domains. To dissect these 158 interactions more rigorously, we carefully analyzed the three segments of the Dk-Helix that face 159 the $\alpha$ C helix in the kinase domain, the disordered AS and the Ct-Helix (Fig. 3a).

Figure 2. The deuterium uptake around the Dk-Helix. (a) The deuterium uptake of selected peptides is plotted and mapped on the LRRK $2_{\text {RCKW }}$ structure. The charts are color coded to the corresponding regions shown. The middle of the Dk-Helix has almost no deuterium uptake suggesting that it is shielded from the solvent. Other peptides that are located at the surface and the activation loop all demonstrate high deuterium uptake. Binding of MLi-2 reduces the deuterium uptake of COR-B-Kinase linker, the $\alpha \mathrm{C}$ helix, and the A-Loop. The uptake is also reduced in peptides that are located around the N-terminal or C-terminal ends of the Dk-Helix. Peptide 1426 - 1449 in the ROC domain is the only peptide that its uptake increases when binding to MLi-2. (b) The relative deuterium exchange for each peptide detected from the $\mathrm{N}$-terminus to the C-terminus of $\mathrm{LRRK} 2_{\mathrm{RKCW}}$ in apo state the kinase (Black) and MLi- 2 bound (red) conditions at $2 \mathrm{~min}$. The arrows indicate the peptides shown in (a)

Figure 3. Characterization of Dk-Helix. (a) The Dk-Helix, the $\alpha \mathrm{C}$ helix and Ct-Helix, and the ROC domain

171 that near the C-terminal end of the Dk-Helix are colored based on the relative fractional uptake. (b) All

172 hydrophobic residues of the Dk-Helix are located on the same side and buried in the COR-B domain 173 while the charged residues that are forming multiple salt bridges with the $\alpha \mathrm{C}$ helix are located on the 174 other side. (c) The surface electrostatic potential of the Dk-Helix. The positively charged N-terminal end 175 of the Dk-Helix is interacting with the Ct-Helix, while the C-terminal end is negatively charged and interacts with the ROC domain. 
residues interact strongly with the $\alpha C$ helix of the kinase domain through electrostatic interactions, locking the $\alpha \mathrm{C}$ helix into an "out" and inactive conformation (Fig. 3c). Typically, in an active kinase the basic residue that lies at the beginning of the $\alpha$ C helix (R1915 in LRRK2) interacts with a phosphate on the A-Loop, which is missing in this structure[14,15]. Our hypothesis is that the N-terminus of the $\alpha \mathrm{C}$ helix very likely becomes "unleashed" when the kinase is in an active conformation.

The N-terminus of the Dk-Helix interacts with the COR-B-Kinase linker that is more solvent exposed and wraps around the $\mathrm{N}$-lobe of the kinase domain (Fig. 3a). This region serves as a "cap" for the N-Lobe of the kinase domain indicating that the conformation and flexibility in particular of the $\mathrm{N}$-lobe appears to be tightly controlled by the linker. The $\mathrm{N}$-terminus of the Dk-Helix, which contains two basic residues (R1771 and K1772), also approaches the end of the Ct-Helix that follows the WD40 domain (Fig. 4a) and contains the last few residues of LRRK2 that are thought to be crucial for kinase activity $[16,17]$. In the LRRK2 ${ }_{R C K W}$ Cryo-EM structure, the terminal three residues (residues 2525-2527) are disordered[10]. In addition, there is a highly flexible loop in the COR-B domain that lies close to the N-lobe of the kinase domain (Fig. 5a). This loop is solvent exposed and also disordered in the LRRK $_{\text {RCKW }}$ structure. Our MD simulations show a dominant interaction of the C-terminal residue, E2527, both the side chain and the $\alpha$-carboxyl group, with R1771 and K1772 at the beginning of the Dk-Helix (Fig. 4b).

Figure 4. Capturing crosstalk that is mediated by the $\mathrm{N}$-and C-termini of the Dk-Helix (a) The Nterminal end of the Dk-Helix is in close proximity to the C-terminal residues of the Ct-Helix which are undiscernible in the cryo-EM structure. (b) GaMD simulations capture the interactions between the DkHelix with the COR-B-Kinase linker and with the C-terminal end of the Ct-Helix (c) In LRRK2 $2_{\mathrm{RCKW}}$, the Dk- 
202

203

204

205

206

207

208

209

210

211

212

213

214

215

216

217

Helix is stably anchored to the $\alpha \mathrm{C}$ helix of the kinase $\mathrm{N}$-lobe. Its $\mathrm{N}$-terminal interacts with the Ct-Helix and the $\mathrm{N}$-lobe of the kinase, while its $\mathrm{C}$-terminal is tethered to the ROC domain and is in close proximity to the A-loop. (d) The C-terminus of the Dk-Helix is anchored to the side chain of R1441 in the ROC domain through W1791. (e) The interaction of E1780 and W1791 with the pathogenic mutation sites R1441 and N1437 on the ROC domain could be captured using GaMD simulations.

Figure 5. The dynamic and of Ct-Helix. (a) The Ct-Helix spans across both the $\mathrm{N}$ - and the C-lobe of the kinase domain with the C-terminus being located in close proximity to the Dk-Helix and the COR-B loop. The Ct-Helix and the according interaction sites are shown and colored by their relative fractional uptake. (b) Showing the surface electrostatic potential of the Ct-Helix. The Ct-Helix docks on the kinase domain through the side that is positively charged while the other side is negatively charged and involved in interactions with the N-terminal domains (NTDs). (c) The deuterium uptake of selected peptides is plotted and mapped on the LRRK2RCKW structure. The CORB-kinase loop and the loop in COR-B domain both show high deuterium uptake $(70 \%-90 \%)$, indicating that they are solvent exposed. And their uptake is reduced in the presence of MLi-2.

\section{Crosstalk between the COR-B domain, the ROC domain, and the Activation Loop in the C-lobe}

\section{of the kinase domain.}

The C-terminus of the Dk-Helix is close to both the A-Loop of the kinase domain and the ROC domain, and in the inactive conformation that is captured by the cryo-EM structure, the tip of this helix is anchored to the ROC domain by the side chain of R1441 which binds to the backbone carbonyl of W1791 and helps to "cap" the Dk-Helix (Fig. 4c and d). The C-terminus of the Dk-Helix also faces the A-Loop of the kinase domain, which is likewise disordered in the LRRK2 $2_{\text {RCKW }}$ Cryo-EM structure (Fig. 3a). We know that this is a critical region because exchange 
224 of R1441 to either C, G or $\mathrm{H}$ is one of the well-documented PD mutations that leads to 225 activation of LRRK2[18]. R1441 as well as N1437, another PD mutation at this interface, are also 226 thought to impair the monomer-dimer cycle of LRRK2 and affect GTPase activity[19].

While a single static conformational state is trapped in the cryo-EM structure, GaMD

228 simulations capture additional potential domain:domain crosstalk that can occur in this region.

229 The simulations suggest, for example, that the side chain of R1441 can also interact with E1790,

230 a residue that is anchored to R1915 in the $\alpha \mathrm{C}$ helix of the kinase domain (Fig. $3 \mathrm{c}$ and $4 \mathrm{e}$ ). This is

231 the residue that would be predicted to interact with the phosphorylation site (P-site) in the A-

232 Loop when the kinase is in an active conformation[14,15]. The dynamics of the Dk-Helix would

233 likely be significantly influenced by PD mutations of R1441 and N1437, as well as Y1699C, which

234 would all, in principle, uncouple the ROC domain from the COR-B domain, and thus enhance

235 potential interactions with the kinase domain that may facilitate activation of LRRK2. Each of

236 these pathogenic mutations would unleash the COR-B domain by distinct mechanisms, leaving

237 it free to communicate with the A-Loop of the kinase domain. Based on our GaMD simulations,

238 multiple residues, such as Q2022, Y2023, and R2026 in the A loop, can potentially interact with

239 the C-terminus of the Dk-Helix (S4 fig.) once it is unleashed from the ROC domain. These

240 interactions, as discussed later, could be important for stabilizing the A-Loop in an extended

241 conformation, which would obviously affect LRRK2 kinase phosphorylation and activation.

242 Capturing the crosstalk between the Ct-Helix and the kinase domain.

243 The structures of LRRK2 reveal a unique helix at the C-terminus that extends from the WD40

244 domain and spans both $\mathrm{N}$ - and $\mathrm{C}$ - lobes of the kinase domain. This Ct-Helix is present in the 
245 inactive LRRK2 $2_{\text {RCKW }}$ cryo-EM structure and is also docked onto the kinase domain in the full-

246 length cryo-EM structure which corresponds to an inactive dimer indicating that it is a very

247 stable helix $[9,10]$,[11]. The combined HDX-MS data captures the dynamic features of the

248 interface of the Ct-Helix with the kinase domain (Fig. 5a). The short segment connecting the

249 kinase and the WD40 domain is embedded between the N-terminus of the Ct-Helix and the C-

250 terminus of the $\alpha \mathrm{E}$ helix in the kinase domain and all show low deuterium uptake suggesting a

251 stable interaction between the WD40 domain and the C-lobe of the kinase domain that is

252 shielded from solvent. The Ct-Helix also interacts with the $\beta 7-\beta 8$ loop in the kinase domain,

253 which is larger in LRRK2 when compared to most kinases (Fig. 5a). The $\alpha$ C- $\beta 4$ loop of the kinase

254 domain, which is an allosteric docking surface for some kinases such as BRAF[20], is almost

255 completely shielded from solvent (S5 Fig.). The Ct-Helix interacts with the kinase domain

256 through both hydrophobic and positively charged residues on one side (Fig. 5b). The mainly

257 negatively charged residues on the other surface could potentially create a binding interface

258 with the N-terminal domains (NTDs) of LRRK2 when it is inhibited. The recently solved cryo-EM

259 structure of full-length LRRK2 revealed how the ANK and LRR domains interact with the Ct-Helix

260 in the inactive state[11]. The charged surface could also be involved in binding or tethering to

261 substrates or activators when the N-terminal non-catalytic domains are "unleashed" from the

262 kinase domain. 
267 domain (Fig. 6a). The two peptides that cover the COR-B-Kinase linker (residue 1840-1861 and

268 residue 1862-1876) have $70 \%$ and $75 \%$ uptake of deuterium at 2 min, respectively, and both

269 show a noticeable decrease in deuterium uptake upon binding of MLi-2. The loop in the COR-B

270 domain, which interacts with the C-terminal tail (residues 1713-1724) also shows reduced

271 uptake (Fig. 5c). These changes in deuterium uptake in response to MLi-2 binding indicate

272 correlated changes in dynamics that include both the kinase and COR-B domains and highlights

273 the crosstalk between the GTPase and kinase domains. Our GaMD simulations capture some of

274 the potential interactions that could occur in this region (Fig. 4b and 6b). C-terminal residue

275 E2527 and the free C-terminal carboxyl moiety are of particular interest as the simulations

276 show how the last five residues can fluctuate between different structural states and form

277 different interactions with the COR-B domain, the N-lobe of the kinase domain and the linker

278 between the COR-B domain and the kinase domain. Two arginine residues, R1723 and R1725, in

279 the long loop of the COR-B domain that is disordered in the cryo-EM structures, form H-bonds

280 with E2527 (Fig. 6b). Another two positively charged residues, R1771 and K1772, at the N-

281 terminal end of the Dk-Helix could stabilize the C-terminal tail (residue 2522-2527) based on

282 the simulations (Fig. 4b). R1866 on the COR-B-Kinase linker can also bind to E2527. Other

283 residues, such as E1899 in the loop that connects the $\beta 2$ and $\beta 3$ strands of the kinase domain,

284 can also interact with R2523 (Fig. 6b). In addition, the two hydroxyl groups from T2524 and

285 S2525 are also capable of forming H-bonds with either the COR-B or the COR-B-Kinase linker.

286 These interactions that appear in different states during the simulations, show how the C-

287 terminal tail can potentially bridge to the COR-B domain and the kinase domain and thereby

288 contribute to the crosstalk between the kinase and GTPase domains. 
Figure 6. Capping of the $\mathbf{N}$-lobe of the kinase. (a) In the LRRK2RCKW cryo-EM structure, the linker from

COR-B to the kinase domain lies over the N-Lobe of the kinase domain. Nearby is a disordered loop from

COR-B and the disordered three terminal residues. The loops, Dk-Helix and the Ct-Helix are colored

based on the relative fractional uptake (b) MD simulations capture potential cross talk between the C-

terminal residues, the COR-B-kinase Loop, and basic residues at the $\mathrm{N}$-terminus of the Dk-Helix.

T2524 near the $\mathrm{N}$-terminus is a known auto-phosphorylation site that can be recognized could in turn affect LRRK2 activity. Also, yet to be resolved is whether 14-3-3 binding would stabilize an active or an inactive dimer.

\section{Capturing the dynamics of the Activation Loop}

As a frame of reference for the LRRK2 AS we show the AS of the cAMP-dependent protein kinase (PKA) when the A-Loop is phosphorylated and the kinase is in a fully closed conformation (Fig. 7a). In PKA the AS begins with the DFG $\psi$ motif and ends with the APE motif, two of the most highly conserved motifs in the protein kinase superfamily[23]. In between these two motifs are the A-Loop and the P+1 Loop. The APE- $\alpha$ F linker that follows the AS, which typically play an important role in docking of substrates and other proteins[13], and should be considered an extended part of the AS. Our HDX-MS results show that the A-Loop and part of 
movie), which is consistent with the fact that the A-Loop and most of the AS are not resolved in the LRRK2 $2_{\text {RCKW }}$ cryo-EM structure (Fig. 7b). The region extending from the APE motif through to the $\alpha \mathrm{F}$ helix is, however, folded, and overlays well with the corresponding region of PKA.

314 Several key residues in this region face out towards the solvent, with the corresponding 315 residues in PKA serving as a docking site for the regulatory subunits (R) (S7 Fig.). Y2050 is also highly conserved in most kinases and it bridges to the backbone residues of this P-site residue when the active kinase is phosphorylated on its A-Loop[24,25] (S8 Fig.). In the cryo-EM structure of LRRK2, this Tyr is not in an active-like conformation. Another interaction in this region is a critical feature that distinguishes the eukaryotic protein kinases (EPKs) from the eukaryotic-like kinases (ELKs), which are their evolutionary precursors[24]. In the ELKs, the A-

321 Loops are short and not dynamic. In addition, the helical domain consisting of the $\alpha \mathrm{G}, \alpha \mathrm{H}$, and $322 \alpha$ l helices is unique to the EPKs. Within this helical domain is a highly conserved Arg between 323 the $\alpha \mathrm{H}$ and the $\alpha \mathrm{l}$ helices. The A-Loop is anchored to this helical domain by a key and highly 324 conserved electrostatic interaction between E2042 in the APE motif of LRRK and R2122 in the $325 \alpha \mathrm{H}$ - $\alpha$ l loop. In the LRRK2 ${ }_{\text {RCKW }}$ cryo-EM structure these two residues are close but not within 326 hydrogen bonding distance; however, the interaction between E2042 and R2122 is captured 327 frequently in the GaMD simulations (S9 Fig.). the AS of active PKA are summarized (PDB: 1ATP). The AS begins with the DFG motif and ends with the 330 APE motif, two of the most highly conserved motifs in the protein kinase superfamily. In between these two motifs are the A-Loop and the P+1 Loop. (b) The AS in the inactive LRRK2RCKW structure is mostly 
missing. The A-Loop phosphate in PKA, pT197, is a red sphere and the corresponding residue in LRRK2 is also a red sphere. Additional P-sites in LRRK2 are shown as black spheres. also contains ATP, the AS is mostly ordered except for 3 residues (Fig. 7c). In this structure the

$\mathrm{P}+1$ loop is ordered in a way that overlays well with PKA. However, the DFG $\psi$ motif region is ordered in a helix that is buttressed up against the $\mathrm{N}$-lobe in contrast to an active conformation where the DFG $\psi$ motif would be fused to a beta strand that binds to the C-lobe (S8 Fig.). Based on PKA, there are at least two docking motifs embedded within the AS. One docking site is created by the outward facing surface of the A-Loop; the other is created by the outward docking surface of the APE- $\alpha \mathrm{F}$ motif. In PKA these two sites are integrated to create a highly dynamic allosteric site that is destroyed by the binding of CAMP (S7 Fig.). In LRRK2, based on our HDX-MS we predict that the A-Loop will reach over to the Dk-Helix while the APE- $\alpha$ F linker will dock onto COR-A. Both states are captured in our GaMD simulations (S10. Fig).

\section{Discussion} well as targeting to different subcellular sites and the transition between monomeric and dimeric states. In an attempt to capture some of the inter-domain crosstalk, we analyzed the cryo-EM LRRK2 $2_{\text {RCKW }}$ structure[10]. This structure, which represents a static snapshot, was used as our starting point. With HDX-MS and GaMD simulations we were able to explore more

352 deeply in domain:domain interfaces and loop dynamics which allowed us to create a dynamic portrait of LRRK2 $2_{\text {RCKW. }}$. Based on the solvent-shielded and solvent-exposed regions, we defined 
354 three rigid bodies, and were able to confirm this domain organization using GaMD simulations

355 (S11 Fig.). The kinase domain is solidly anchored to the WD40 domain as well as the Ct-Helix

356 that extends from the WD40 domain. This explains why it has not been possible to create a

357 stable isolated kinase domain for LRRK2. The catalytically inert COR domain is comprised of two

358 subdomains, referred to as the COR-A and COR-B domains, joined by a flexible linker. The COR-

359 A domain is firmly anchored to the ROC domain so that these two subdomains also move as a

rigid body while the COR-B domain functions as a separate rigid body that communicates with

both the kinase domain and the ROC domain as well as with the C-terminus (residues 2525-

2527). As predicted by Watanabe et al., this highly dynamic COR-B domain is the major

mediator of crosstalk between the kinase domain and the ROC domain in the active dimer[9].

GaMD show that domain motions are also embedded within the kinase domain. The

kinase domain, for example, toggles between active and inactive states that correlate with

opening and closing of the catalytic cleft (Fig. 8 and S12 Fig.). MLi-2, a type I kinase inhibitor,

367 locks the kinase domain into a closed and active-like conformation while a type II inhibitor is

368 hypothesized to lock the kinase domain into an open conformation[10]. The structure of

369 monomeric LRRK2 $2_{\text {RCKW }}$ serves as a model for the inactive kinase while the full-length I2020T

370 LRRK2 mutant docked onto microtubules in a helical manner represents an active dimer[9].

371 Opening and closing of the kinase cleft, where the $\mathrm{N}$ - and C-lobes move as rigid bodies, is

372 determined by the flexibility of the N-Lobe and its ability to communicate with the C-lobe. In its

373 inactive state, it is locked into an open conformation by the two flanking helices, the Dk-Helix in

374 COR-B domain and the Ct-Helix as well as by an unusual DFG $\psi$ motif in LRRK2, where the highly

375 conserved Phe is replaced with Tyr (DYG $\psi)$ [8]. PD mutations that lead to activation obviously 
376 alter the equilibrium between the active and inactive states. We showed previously how the

377 two PD mutations in the kinase domain (G2019S and I2020T) unleash the inhibitory NTDs, and

378 with our HDX-MS analysis of the kinase domain we showed how the disordered region

379 surrounding the AS becomes more ordered by the binding of MLi-2[12].

In addition to mediating crosstalk between the kinase domain and the GTPase (ROC)

domain, the COR-B domain also controls dimerization of the active kinase $[9,11]$. Embedded

within COR-B are two domain:domain interfaces (Fig. 8). The Dk-Helix interface communicates

directly with the $\alpha C$ helix in the kinase domain while the COR-B:ROC interface is sensitive to the

mutations (R1441 and N1437 in the ROC domain and Y1699 in COR-B domain) also unleash the

inhibitory NTDs by destabilizing this COR-B:ROC interface[8,26]. One final mutation at this

Figure 8. The interfaces in compact or extend conformation of LRRK2 $\mathbf{R C K W}_{\text {. }}$ (a) The Dk-Helix and Ct-Helix

392 are highlighted on the cryo-EM structure of LRRK2RCKW. The pathogenic mutations N1437, R1441 and 
COR-B domain remain intact when the COR-A domain moves away from the C-lobe of the kinase in the extended conformation. and are perturbed by mutations that make LRRK2 a risk factor for PD.

\section{Materials and Methods}

\section{Hydrogen-deuterium exchange mass spectrometry}

414 LRRK2 $2_{\text {RCKW }}$ proteins were expressed and purified from Sf9 cell[12]. Hydrogen/deuterium

415 exchange mass spectrometry (HDX-MS) was performed using a Waters Synapt G2Si equipped

416 with nanoACQUITY UPLC system with H/DX technology and a LEAP autosampler. The LRRK2 $2_{\text {RCKW }}$

417 concentration was $5 \mu \mathrm{M}$ in LRRK2 buffer containing: $20 \mathrm{mM} \mathrm{HEPES} / \mathrm{NaOH} \mathrm{pH}$ 7.4, $800 \mathrm{mM} \mathrm{NaCl}$, 
measured in LRRK2 buffer in the presence and absence of the kinase inhibitor MLi-2 (50 $\mu \mathrm{M})$. For each deuteration time, $4 \mu \mathrm{L}$ complex was equilibrated to $25{ }^{\circ} \mathrm{C}$ for $5 \mathrm{~min}$ and then mixed with $56 \mu \mathrm{L} \mathrm{D} \mathrm{D}_{2} \mathrm{O}$ LRRK2 buffer for $0,0.5,1$ or $2 \mathrm{~min}$. The exchange was quenched with an equal volume of quench solution ( $3 \mathrm{M}$ guanidine, $0.1 \%$ formic acid, $\mathrm{pH} 2.66$ ). The quenched sample $(50 \mu \mathrm{L})$ was injected into the sample loop, followed by digestion on an in-line pepsin column (immobilized pepsin, Pierce, Inc.) at $15^{\circ} \mathrm{C}$. The resulting peptides were captured on a BEH C18 Vanguard pre-column, separated by analytical chromatography (Acquity UPLC BEH C18, $1.7 \mu \mathrm{M}$, $1.0 \times 50 \mathrm{~mm}$, Waters Corporation) using a $7-85 \%$ acetonitrile gradient in $0.1 \%$ formic acid over $7.5 \mathrm{~min}$, and electrosprayed into the Waters SYNAPT G2Si quadrupole time-of-flight mass spectrometer. The mass spectrometer was set to collect data in the Mobility, ESI+ mode; mass acquisition range of $200-2,000(\mathrm{~m} / \mathrm{z})$; scan time $0.4 \mathrm{~s}$. Continuous lock mass correction was accomplished with infusion of leu-enkephalin $(\mathrm{m} / \mathrm{z}=556.277$ ) every $30 \mathrm{~s}$ (mass accuracy of $1 \mathrm{ppm}$ for calibration standard). For peptide identification, the mass spectrometer was set to collect data in $\mathrm{MS}^{\mathrm{E}}, \mathrm{ESI}+$ mode instead.

The peptides were identified from triplicate $\mathrm{MS}^{\mathrm{E}}$ analyses of $10 \mu \mathrm{M} \operatorname{LRRK} 2_{\mathrm{RCKW}}$, and data were analyzed using PLGS 3.0 (Waters Corporation). Peptide masses were identified using a minimum number of 250 ion counts for low energy peptides and 50 ion counts for their fragment ions. The peptides identified in PLGS were then analyzed in DynamX 3.0 (Waters Corporation) using a cut-off score of 6.5 , error tolerance of $5 \mathrm{ppm}$ and requiring that the peptide be present in at least 2 of the 3 identification runs. The peptides reported on the coverage maps are those from which data were obtained. The relative deuterium uptake for each peptide was calculated by comparing the centroids of the mass envelopes of the 
441 deuterated samples vs. the undeuterated controls[30]. For all HDX-MS data, at least 2 biological

442 replicates were analyzed each with 3 technical replicates. Data are represented as mean values

443 +/- SEM of 3 technical replicates due to processing software limitations, however the LEAP

444 robot provides highly reproducible data for biological replicates. The deuterium uptake was

445 corrected for back-exchange using a global back exchange correction factor (typically 25\%)

446 determined from the average percent exchange measured in disordered termini of various

447 proteins[31]. Deuterium uptake plots were generated in DECA (github.com/komiveslab/DECA)

448 and the data are fitted with an exponential curve for ease of viewing[32].

449 Gaussian accelerated Molecular Dynamics (GaMD) simulation

450 The LRRK $2_{\text {RCKW }}$ model for simulations were prepared based on the reported LRRK $2_{\text {RCKW }}$ structure

451 (PDB: 6VP6) using Modeller to model the missing loops[33]. The Protein Preparation Wizard

452 was used to build missing sidechains and model charge states of ionizable residues at neutral

$453 \mathrm{pH}$. Hydrogens and counter ions were added and the models were solvated in a cubic box of

454 TIP4P-EW water[34] and $150 \mathrm{mM} \mathrm{KCl}$ with a $10 \AA$ buffer in AMBER tools D.A. Case, 2016 \#731\}.

455 AMBER16 was used for energy minimization, heating, and equilibration steps, using the CPU

456 code for minimization and heating and GPU code for equilibration. Parameters from the Bryce

457 AMBER parameter database were used for phosphoserine and phosphothreonine[35]. Systems

458 were minimized by 1000 steps of hydrogen-only minimization, 2000 steps of solvent

459 minimization, 2000 steps of ligand minimization, 2000 steps of side-chain minimization, and

4605000 steps of all-atom minimization. Systems were heated from $0 \mathrm{~K}$ to $300 \mathrm{~K}$ linearly over

461200 ps with $2 \mathrm{fs}$ time-steps and $10.0 \mathrm{kcal} / \mathrm{mol} / \AA$ p position restraints on protein. Temperature

462 was maintained by the Langevin thermostat. Constant pressure equilibration with an $8 \AA$ non- 
463 bonded cut-off with particle mesh Ewald was performed with $300 \mathrm{ps}$ of protein and peptide

464 restraints followed by 900 ps of unrestrained equilibration. Gaussian accelerated MD (GaMD)

465 was used on GPU enabled AMBER16 to enhance conformational sampling [36]. GaMD applies a

466 Gaussian distributed boost energy to the potential energy surface to accelerate transitions

467 between meta-stable states while allowing accurate reweighting with cumulant expansion.

468 Both dihedral and total potential acceleration were used simultaneously. Potential statistics

469 were collected for 2 ns followed by 2 ns of GaMD during which boost parameters were updated

470 for each simulation. Each GaMD simulation was equilibrated for 10 ns. For each construct 10

471 independent replicates of 200 ns of GaMD simulation were run in the NVT ensemble, for an

472 aggregate of $2.0 \mu$ s of accelerated MD.

473 Acknowledgements

474 This work was supported by Michael J. Fox Foundation Grant 11425

475 (https://www.michaeljfox.org/) (to S.S.T., and F.W.H.), and Ruth L. Kirschstein National

476 Research Service Award NIH/National Cancer Institute T32 CA009523 (to P.C.A.). JTM was

477 supported by an Otto-Braun Fund Predoctoral Fellowship (B. Braun Melsungen AG). S.M. and

478 S.K. are grateful for support from the Deutsche Forschungsgemeinschaft (DFG) (HE 1818/11)

479 and Structural Genomics Consortium (SGC), a registered charity that receives funds from

480 AbbVie, Bayer Pharma AG, Boehringer Ingelheim, Canada Foundation for Innovation, Eshelman

481 Institute for Innovation, Genome Canada, Innovative Medicines Initiative (875510), Janssen,

482 Merck KGaA Darmstadt Germany, Merck Sharp and Dohme (MSD), Novartis Pharma AG,

483 Ontario Ministry of Economic Development and Innovation. The Synapt G2Si HD/X mass

484 spectrometer was obtained from shared instrumentation NIH Grant S10 OD016234 (to S.S.). 
485 The funders had no role in study design, data collection and analysis, decision to publish, or

486 preparation of the manuscript.

487 Author Contributions

488 J.-H.W., P.C.A., S.M., F.W.H., and S.S.T. designed research; J.-H.W., P.C.A., and S.M. performed

489 research; J.-H.W., P.C.A., S.H.S., R.L., J.W., J. T. M., P. K.-S., S.M., S. K., F.W.H., and S.S.T.

490 analyzed data; and J.-H.W., P.C.A., R.L., J.W., J. T. M., P. K.-S., F.W.H., and S.S.T. wrote the paper.

491 All author reviews the manuscript.

492 Competing Interests statement

493 The authors have no competing interests. 


\section{References}

1. Benitez BA, Davis AA, Jin SC, Ibanez L, Ortega-Cubero S, et al. (2016) Resequencing analysis of five Mendelian genes and the top genes from genome-wide association studies in Parkinson's Disease. Mol Neurodegener 11: 29.

2. Mata IF, Wedemeyer WJ, Farrer MJ, Taylor JP, Gallo KA (2006) LRRK2 in Parkinson's disease: protein domains and functional insights. Trends Neurosci 29: 286-293.

3. Tomkins JE, Dihanich S, Beilina A, Ferrari R, llacqua N, et al. (2018) Comparative Protein Interaction Network Analysis Identifies Shared and Distinct Functions for the Human ROCO Proteins. Proteomics 18: e1700444.

4. Sheng Z, Zhang S, Bustos D, Kleinheinz T, Le Pichon CE, et al. (2012) Ser1292 autophosphorylation is an indicator of LRRK2 kinase activity and contributes to the cellular effects of PD mutations. Sci Transl Med 4: 164ra161.

5. Nguyen AP, Moore DJ (2017) Understanding the GTPase Activity of LRRK2: Regulation, Function, and Neurotoxicity. Adv Neurobiol 14: 71-88.

6. West AB, Moore DJ, Choi C, Andrabi SA, Li X, et al. (2007) Parkinson's disease-associated mutations in LRRK2 link enhanced GTP-binding and kinase activities to neuronal toxicity. Hum Mol Genet 16: 223-232.

7. Cookson MR (2017) Mechanisms of Mutant LRRK2 Neurodegeneration. Adv Neurobiol 14: 227-239.

8. Schmidt SH, Knape MJ, Boassa D, Mumdey N, Kornev AP, et al. (2019) The dynamic switch mechanism that leads to activation of LRRK2 is embedded in the DFGpsi motif in the kinase domain. Proc Natl Acad Sci U S A.

9. Watanabe R, Buschauer R, Bohning J, Audagnotto M, Lasker K, et al. (2020) The In Situ Structure of Parkinson's Disease-Linked LRRK2. Cell 182: 1508-1518 e1516.

10. Deniston CK, Salogiannis J, Mathea S, Snead DM, Lahiri I, et al. (2020) Structure of LRRK2 in Parkinson's disease and model for microtubule interaction. Nature.

11. Myasnikov A, Zhu H, Hixson P, Xie B, Yu K, et al. (2021) Structural analysis of the full-length human LRRK2. Cell.

12. Schmidt SH, Weng JH, Aoto PC, Boassa D, Mathea S, et al. (2021) Conformation and dynamics of the kinase domain drive subcellular location and activation of LRRK2. Proc Natl Acad Sci U S A 118.

13. Taylor SS, Kornev AP (2011) Protein kinases: evolution of dynamic regulatory proteins. Trends Biochem Sci 36: 65-77.

14. Nolen B, Taylor S, Ghosh G (2004) Regulation of protein kinases; controlling activity through activation segment conformation. Mol Cell 15: 661-675.

15. Johnson LN, Noble ME, Owen DJ (1996) Active and inactive protein kinases: structural basis for regulation. Cell 85: 149-158.

16. Kett LR, Boassa D, Ho CC, Rideout HJ, Hu J, et al. (2012) LRRK2 Parkinson disease mutations enhance its microtubule association. Hum Mol Genet 21: 890-899.

17. Rudenko IN, Kaganovich A, Hauser DN, Beylina A, Chia R, et al. (2012) The G2385R variant of leucinerich repeat kinase 2 associated with Parkinson's disease is a partial loss-of-function mutation. Biochem J 446: 99-111.

18. Haugarvoll K, Wszolek ZK (2009) Clinical features of LRRK2 parkinsonism. Parkinsonism Relat Disord 15 Suppl 3: S205-208.

19. Huang X, Wu C, Park Y, Long X, Hoang QQ, et al. (2019) The Parkinson's disease-associated mutation $\mathrm{N} 1437 \mathrm{H}$ impairs conformational dynamics in the G domain of LRRK2. FASEB J 33: 4814-4823. 
20. Hu J, Stites EC, Yu H, Germino EA, Meharena HS, et al. (2013) Allosteric activation of functionally asymmetric RAF kinase dimers. Cell 154: 1036-1046.

21. Pungaliya PP, Bai Y, Lipinski K, Anand VS, Sen S, et al. (2010) Identification and characterization of a leucine-rich repeat kinase 2 (LRRK2) consensus phosphorylation motif. PLoS One 5: e13672.

22. Manschwetus JT, Wallbott M, Fachinger A, Obergruber C, Pautz S, et al. (2020) Binding of the Human 14-3-3 Isoforms to Distinct Sites in the Leucine-Rich Repeat Kinase 2. Front Neurosci 14: 302.

23. Johnson DA, Akamine P, Radzio-Andzelm E, Madhusudan M, Taylor SS (2001) Dynamics of cAMPdependent protein kinase. Chem Rev 101: 2243-2270.

24. Taylor SS, Keshwani MM, Steichen JM, Kornev AP (2012) Evolution of the eukaryotic protein kinases as dynamic molecular switches. Philos Trans R Soc Lond B Biol Sci 367: 2517-2528.

25. Krupa A, Preethi G, Srinivasan N (2004) Structural modes of stabilization of permissive phosphorylation sites in protein kinases: distinct strategies in Ser/Thr and Tyr kinases. J Mol Biol 339: 1025-1039.

26. Taylor SS, Kaila-Sharma P, Weng JH, Aoto P, Schmidt SH, et al. (2020) Kinase Domain Is a Dynamic Hub for Driving LRRK2 Allostery. Front Mol Neurosci 13: 538219.

27. Hui KY, Fernandez-Hernandez H, Hu J, Schaffner A, Pankratz N, et al. (2018) Functional variants in the LRRK2 gene confer shared effects on risk for Crohn's disease and Parkinson's disease. Sci Transl Med 10.

28. Deng J, Lewis PA, Greggio E, Sluch E, Beilina A, et al. (2008) Structure of the ROC domain from the Parkinson's disease-associated leucine-rich repeat kinase 2 reveals a dimeric GTPase. Proc Natl Acad Sci U S A 105: 1499-1504.

29. Prior IA, Lewis PD, Mattos C (2012) A comprehensive survey of Ras mutations in cancer. Cancer Res 72: 2457-2467.

30. Wales TE, Fadgen KE, Gerhardt GC, Engen JR (2008) High-speed and high-resolution UPLC separation at zero degrees Celsius. Anal Chem 80: 6815-6820.

31. Ramsey KM, Dembinski HE, Chen W, Ricci CG, Komives EA (2017) DNA and IkappaBalpha Both Induce Long-Range Conformational Changes in NFkappaB. J Mol Biol 429: 999-1008.

32. Lumpkin RJ, Komives EA (2019) DECA, A Comprehensive, Automatic Post-processing Program for HDX-MS Data. Mol Cell Proteomics 18: 2516-2523.

33. Sali A, Blundell TL (1993) Comparative protein modelling by satisfaction of spatial restraints. J Mol Biol 234: 779-815.

34. Horn HW, Swope WC, Pitera JW, Madura JD, Dick TJ, et al. (2004) Development of an improved foursite water model for biomolecular simulations: TIP4P-Ew. J Chem Phys 120: 9665-9678.

35. Homeyer N, Horn AH, Lanig $\mathrm{H}$, Sticht $\mathrm{H}$ (2006) AMBER force-field parameters for phosphorylated amino acids in different protonation states: phosphoserine, phosphothreonine, phosphotyrosine, and phosphohistidine. J Mol Model 12: 281-289.

36. Miao Y, Feher VA, McCammon JA (2015) Gaussian Accelerated Molecular Dynamics: Unconstrained Enhanced Sampling and Free Energy Calculation. J Chem Theory Comput 11: 3584-3595. 


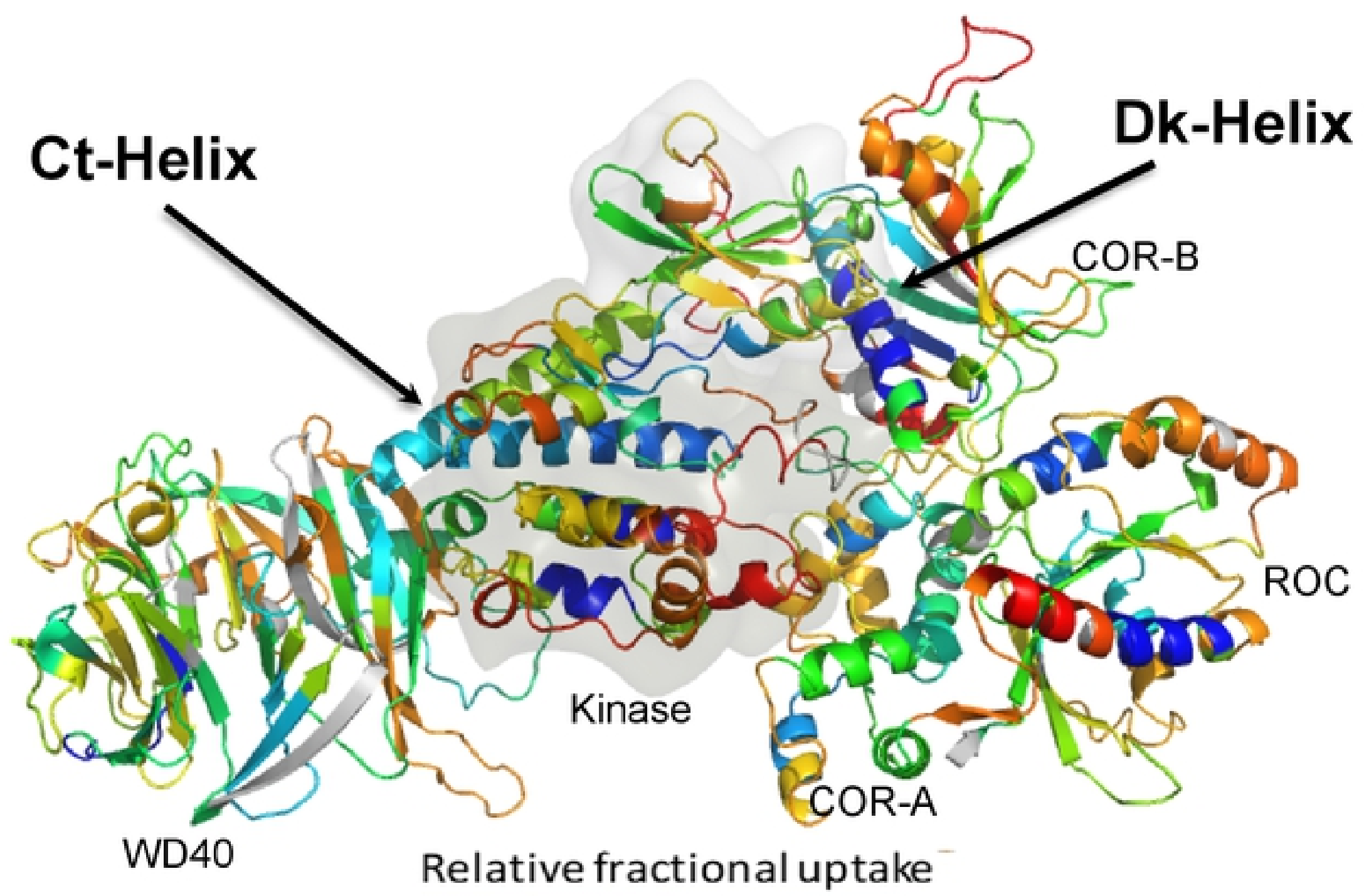

b

bioRxiv preprint doi: https://doi.org/10.1101/2021.09.29.462278; this version posted September 29, 2021. The copyright holder for this preprin

$90 \%$
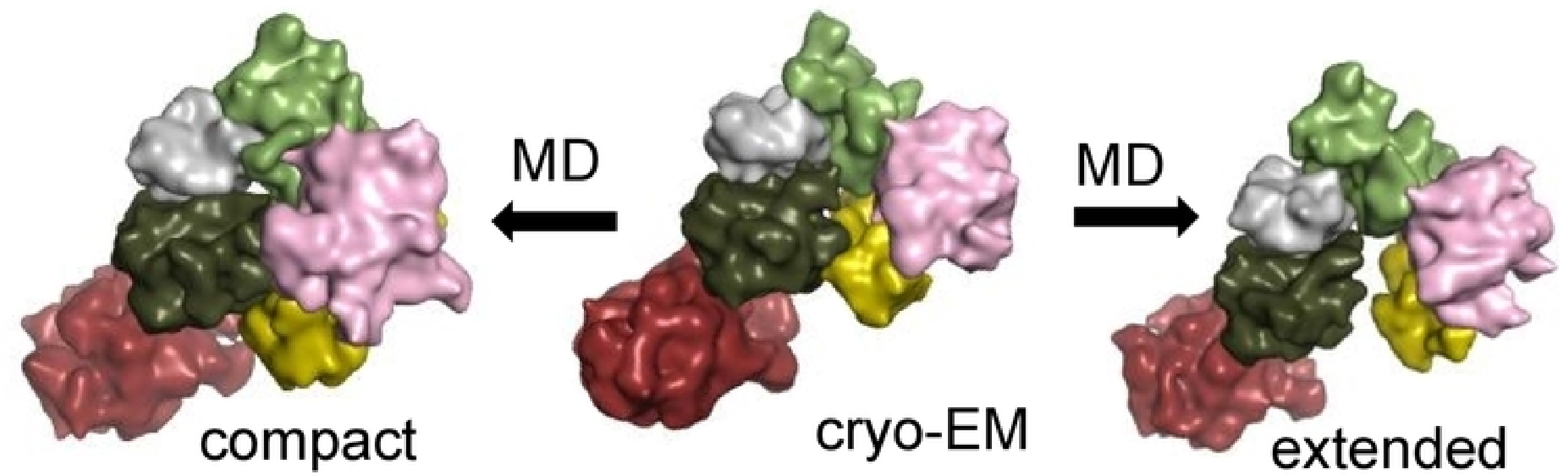

1330

1670

186819512141

2527

\section{ROC COR-A COR-B Kinase}

WD40

Figure 1. The overall dynamic of LRRK2 $_{\text {RCKW }}$. (a) The relative deuterium uptake after 2 min deuterium exposure is color-coded mapped on the LRRK $2_{\mathrm{RCKW}}$ model. Grey color indicates no deuterium uptake information. The surface of the kinase domain is shown in gray. The highly protected Dk-Helix and the Ct-Helix located at the back of the kinase domain are labelled. (B) Snapshot of LRRK2 $2_{\text {RCKW }}$ in the MD simulation. The surface of each domain is shown in different colors. Left represents the compact architecture of $L R R K 2_{R C K W}$. The middle is the structure from cryo-EM. The right represents one of the extended states of LRRK2 $2_{\mathrm{RCKW}}$. 
a

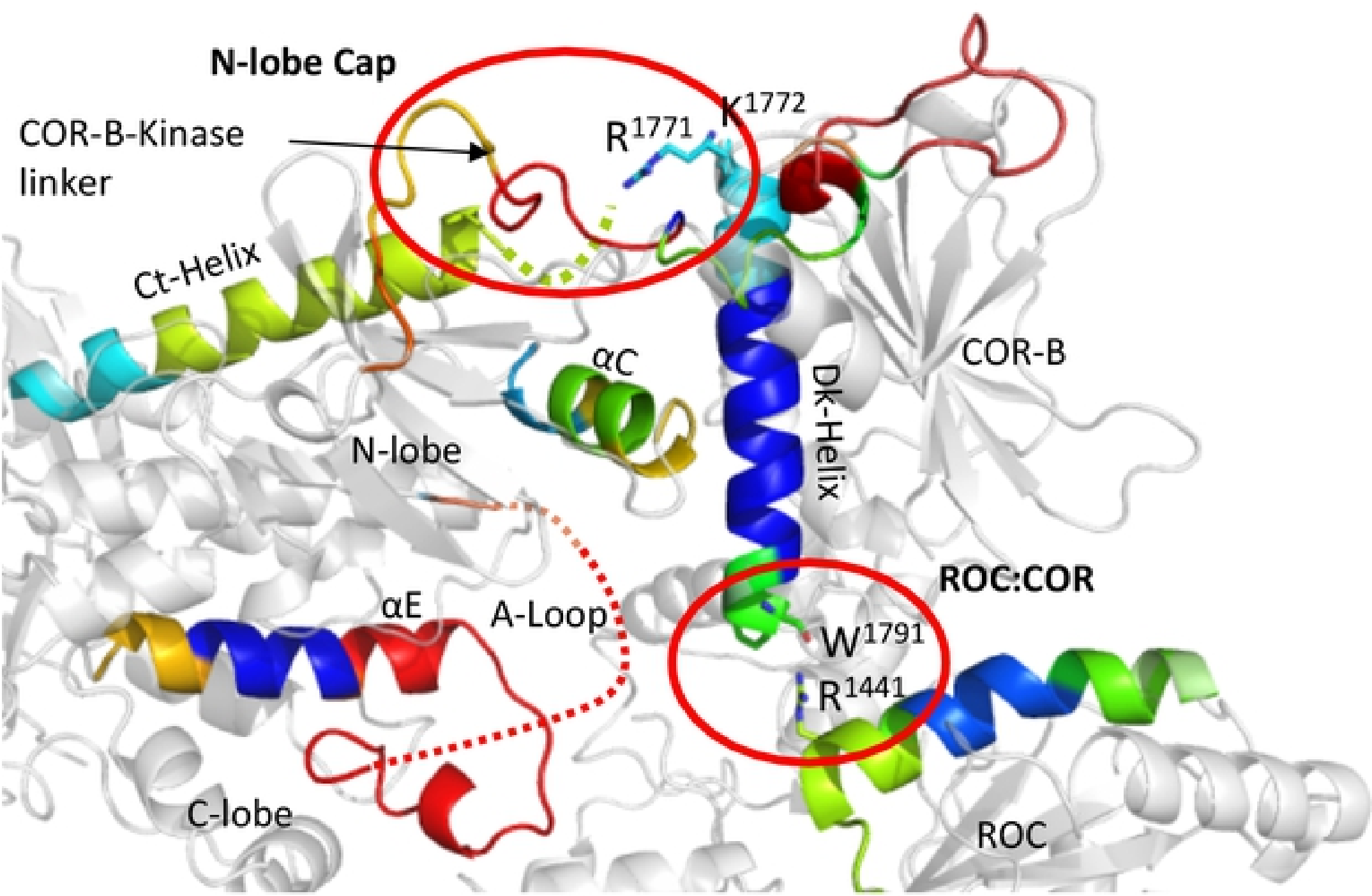

Relative fractional uptake

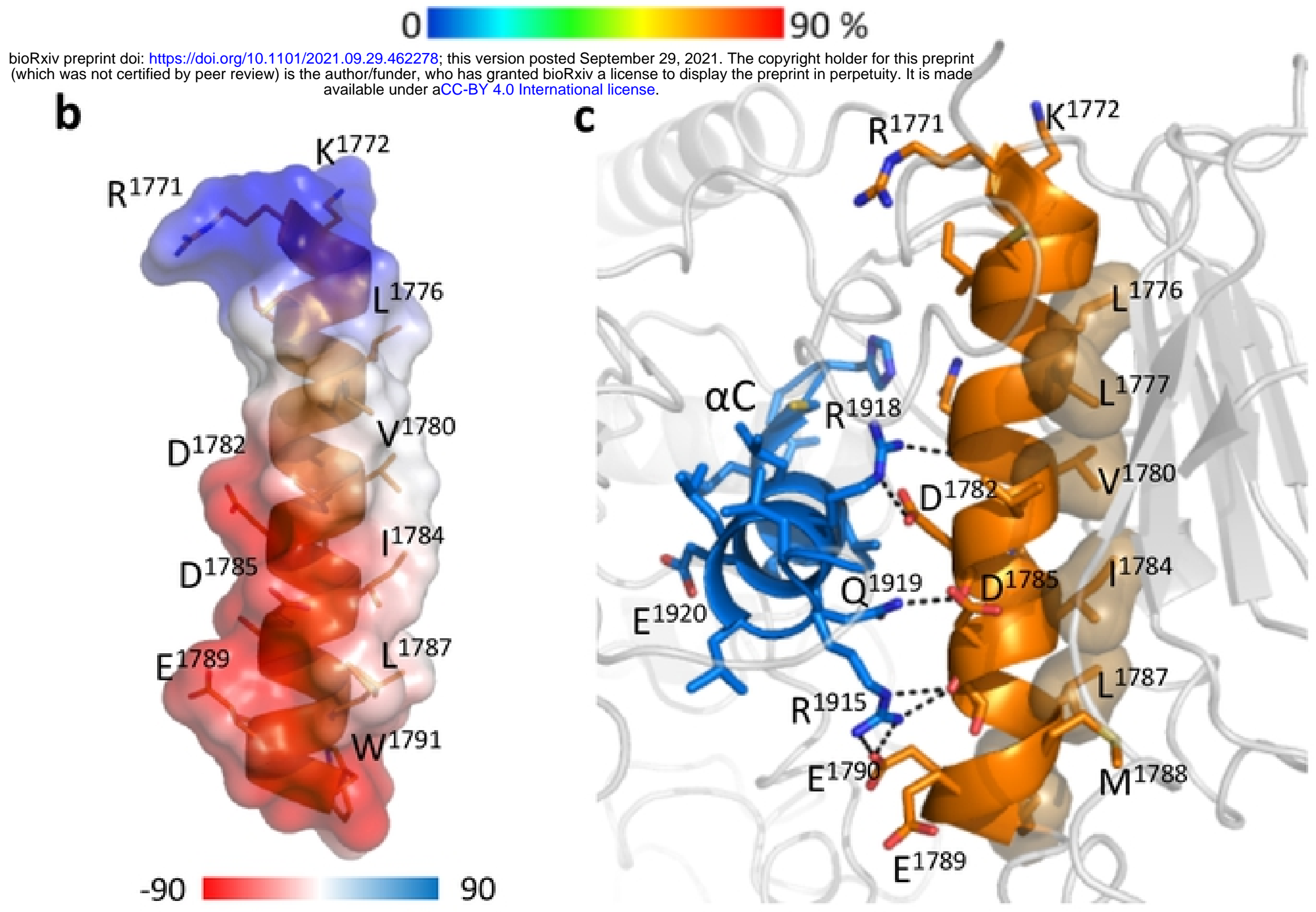

Figure 3. Characterization of Dk-Helix. (a) The Dk-Helix, the $\alpha \mathrm{C}$ helix and Ct-Helix, and the ROC domain that near the C-terminal end of the Dk-Helix are colored based on the relative fractional uptake. (b) All hydrophobic residues of the Dk-Helix are located on the same side and buried in the COR-B domain while the charged residues that are forming multiple salt bridges with the $\alpha \mathrm{C}$ helix are located on the other side. (c) The surface electrostatic potential of the Dk-Helix. The positively charged $\mathrm{N}$-terminal end of the Dk-Helix is interacting with the Ct-Helix, while the C-terminal end is negatively charged and interacts with the ROC domain. 


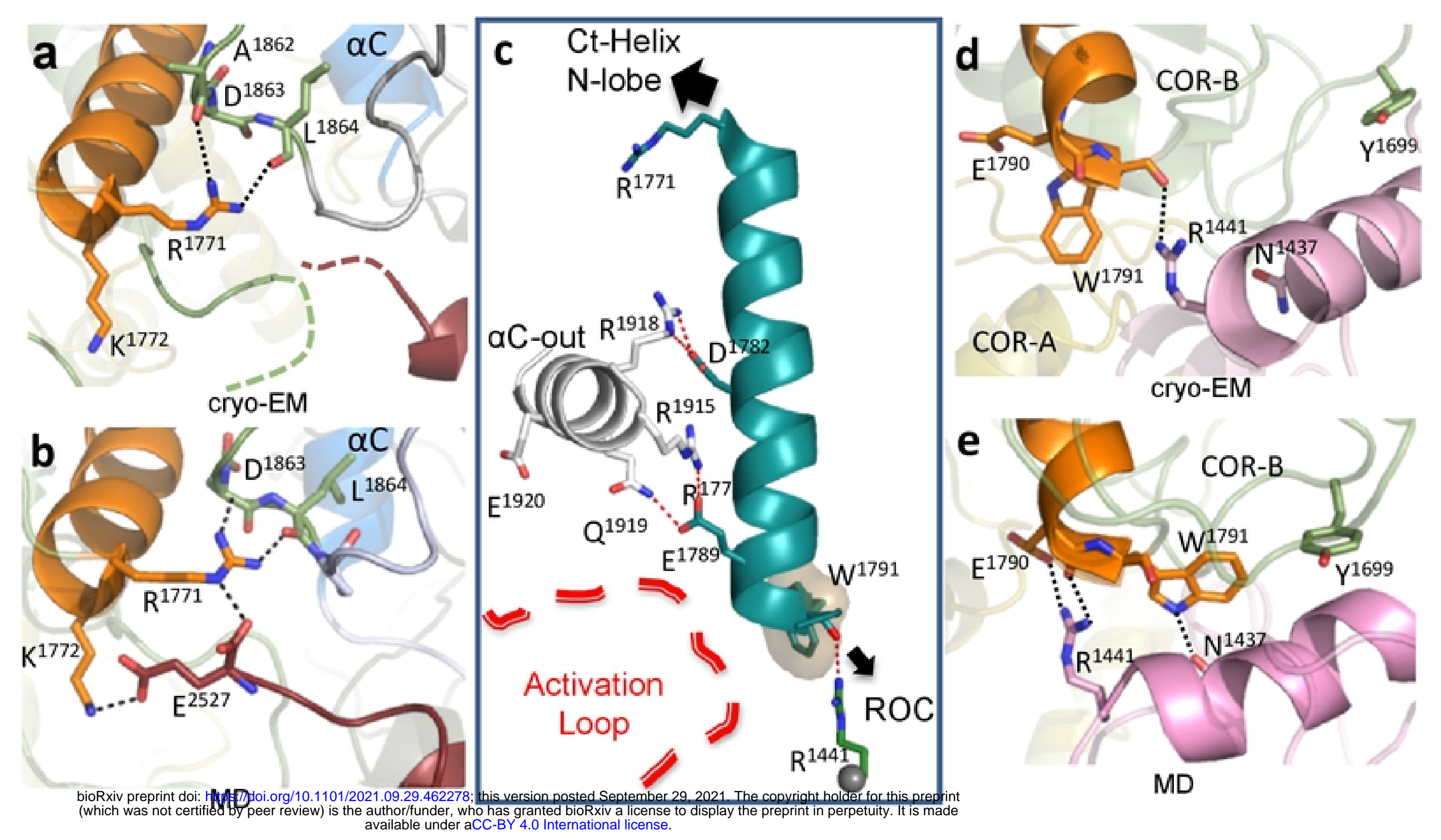

Figure 4. Capturing crosstalk that is mediated by the N-and C-termini of the Dk-Helix (a) The Nterminal end of the Dk-Helix is in close proximity to the C-terminal residues of the Ct-Helix which are undiscernible in the cryo-EM structure. (b) GaMD simulations capture the interactions between the DkHelix with the COR-B-Kinase linker and with the C-terminal end of the Ct-Helix (c) In LRRK2 ${ }_{\text {RCKW }}$, the DkHelix is stably anchored to the $\alpha \mathrm{C}$ helix of the kinase $\mathrm{N}$-lobe. Its $\mathrm{N}$-terminal interacts with the Ct-Helix and the $\mathrm{N}$-lobe of the kinase, while its $\mathrm{C}$-terminal is tethered to the ROC domain and is in close proximity to the A-loop. (d) The C-terminus of the Dk-Helix is anchored to the side chain of R1441 in the ROC domain through W1791. (e) The interaction of E1780 and W1791 with the pathogenic mutation sites R1441 and N1437 on the ROC domain could be captured using GaMD simulations. 


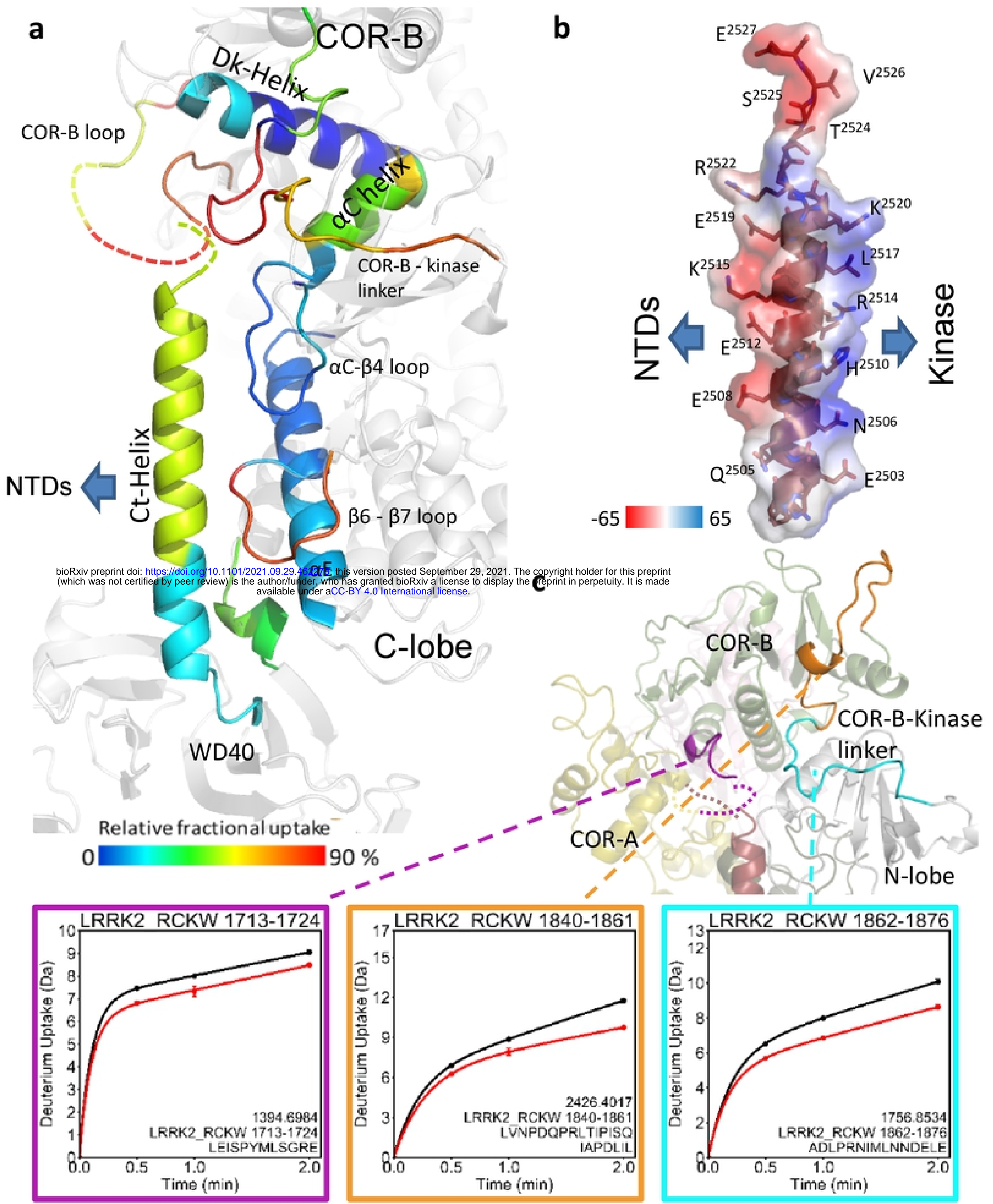

Figure 5. The dynamic and of Ct-Helix. (a) The Ct-Helix spans across both the $\mathrm{N}$ - and the C-lobe of the kinase domain with the C-terminus being located in close proximity to the Dk-Helix and the COR-B loop. The Ct-Helix and the according interaction sites are shown and colored by their relative fractional uptake. (b) Showing the surface electrostatic potential of the Ct-Helix. The Ct-Helix docks on the kinase domain through the side that is positively charged while the other side is negatively charged and involved in interactions with the N-terminal domains (NTDs). (c) The deuterium uptake of selected peptides is plotted and mapped on the LRRK2RCKW structure. The CORB-kinase loop and the loop in COR-B domain both show high deuterium uptake (70\%-90\%), indicating that they are solvent exposed. And their uptake is reduced in the presence of $\mathrm{MLi}-2$. 
a

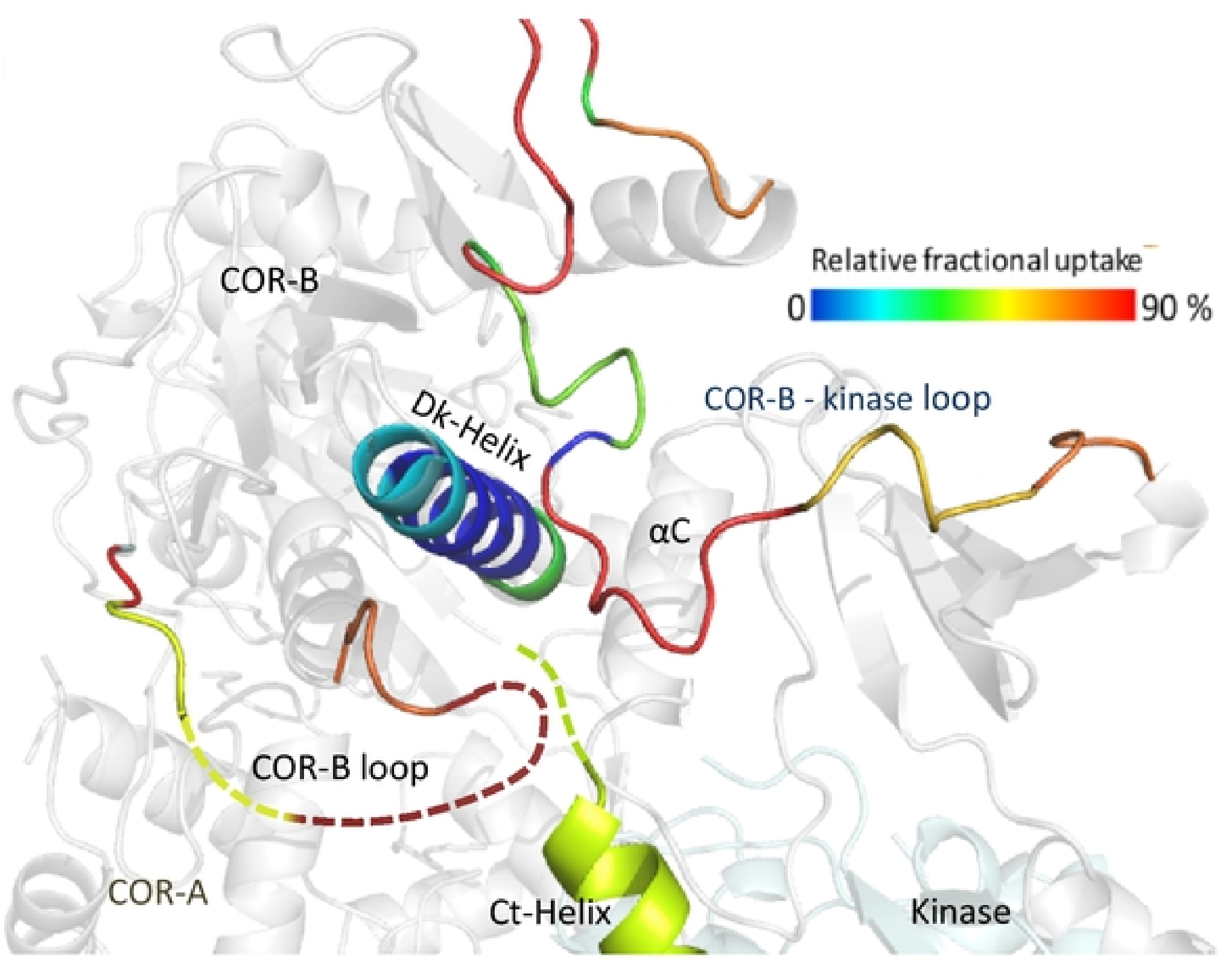

bioRxiv preprint doi: https://doi.org/10.1101/2021.09.29.462278; this version posted September 29, 2021. The copyright holder for this preprint

(which was not certified by peer review) is the author/funder, who has granted bioRxiv a license to display the preprint in perpetuity. It is made

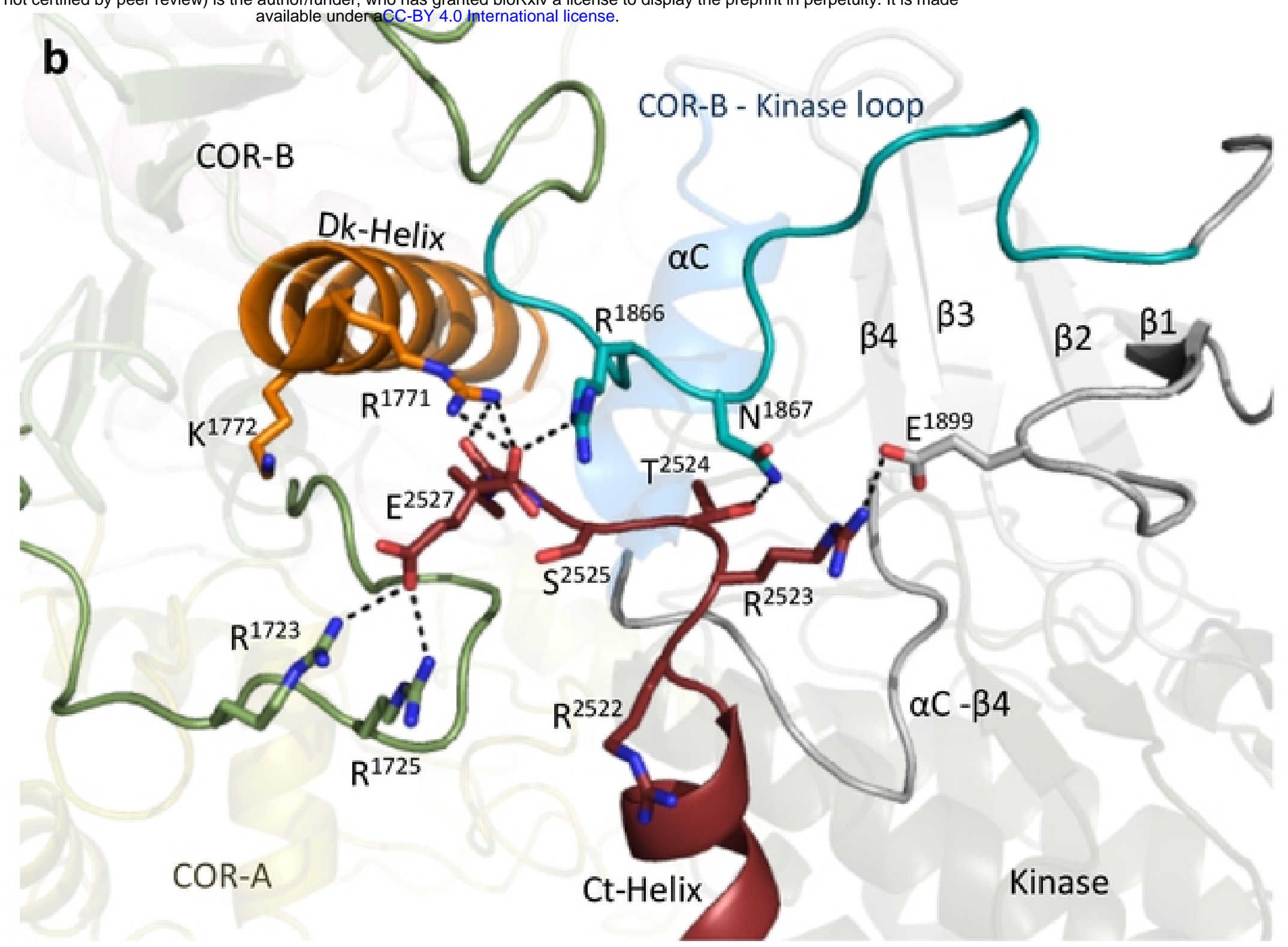

Figure 6. Capping of the $\mathrm{N}$-lobe of the kinase. (a) In the LRRK2RCKW cryo-EM structure, the linker from COR-B to the kinase domain lies over the N-Lobe of the kinase domain. Nearby is a disordered loop from COR-B and the disordered three terminal residues. The loops, Dk-Helix and the Ct-Helix are colored based on the relative fractional uptake (b) MD simulations capture potential cross talk between the C-terminal residues, the COR-B-kinase Loop, and basic residues at the $\mathrm{N}$-terminus of the Dk-Helix. 
a

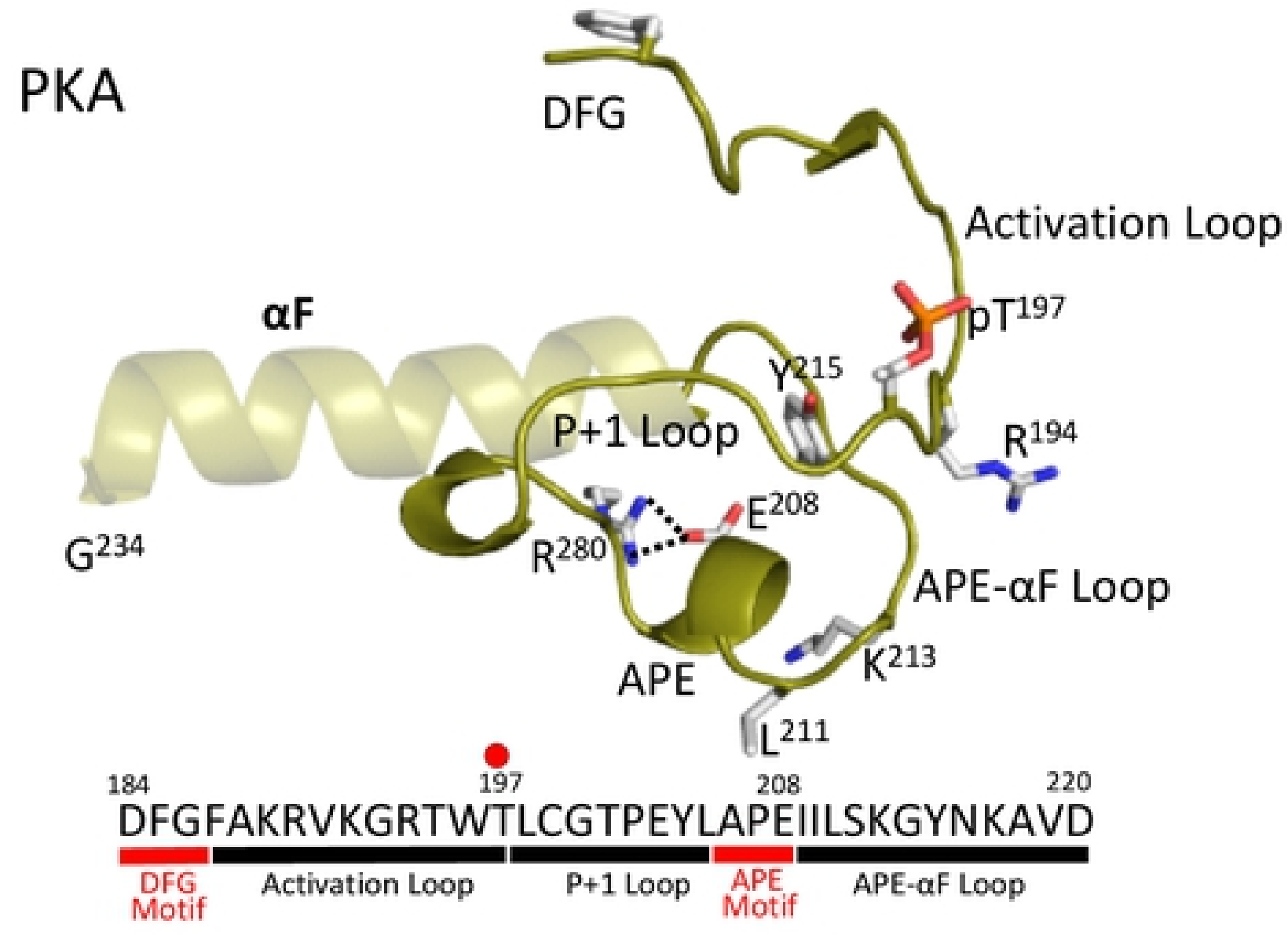

b

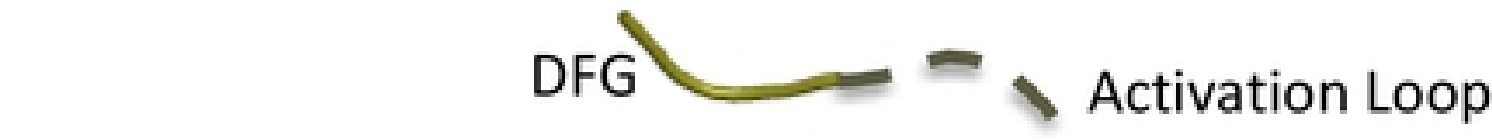

LRRK2 $2_{\text {RCKW }}$

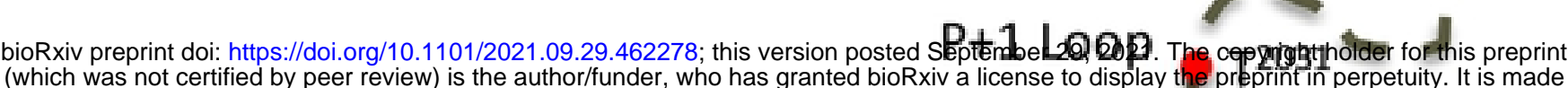

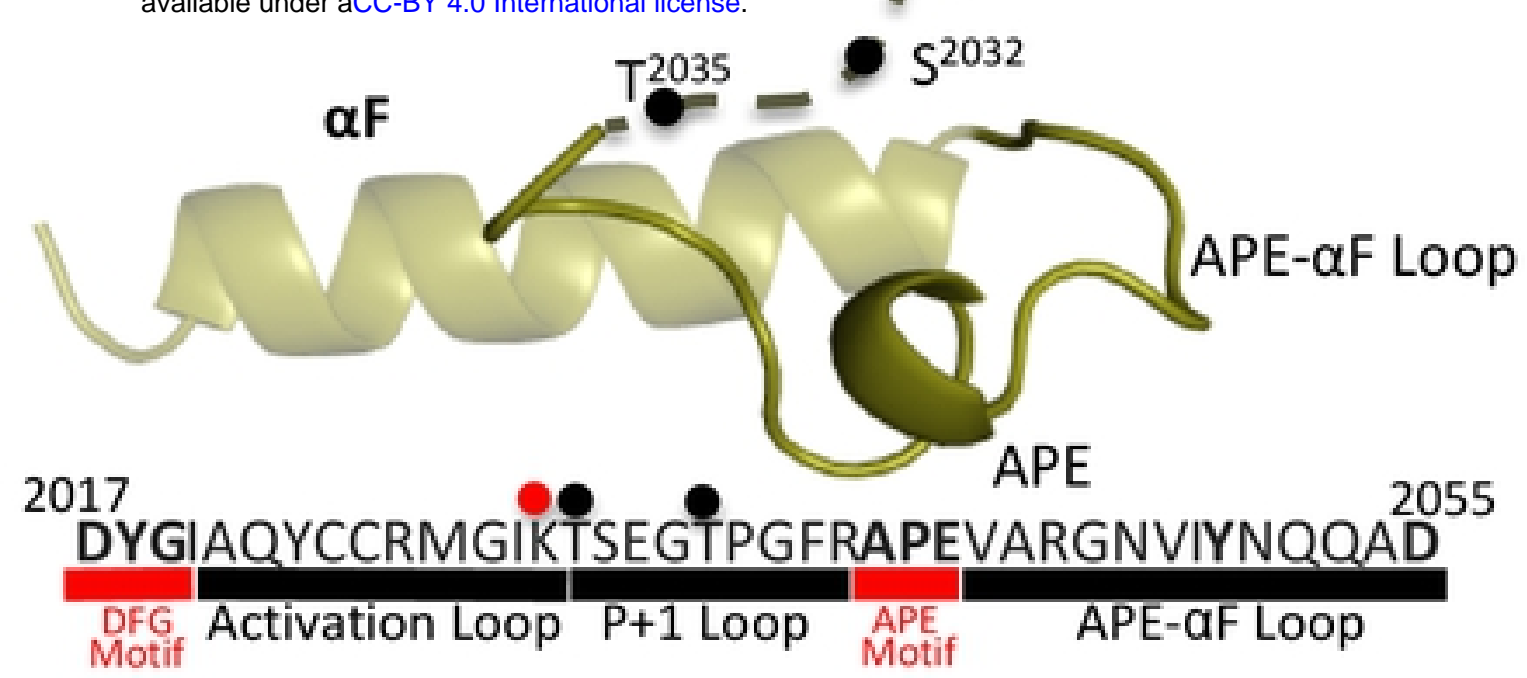

C
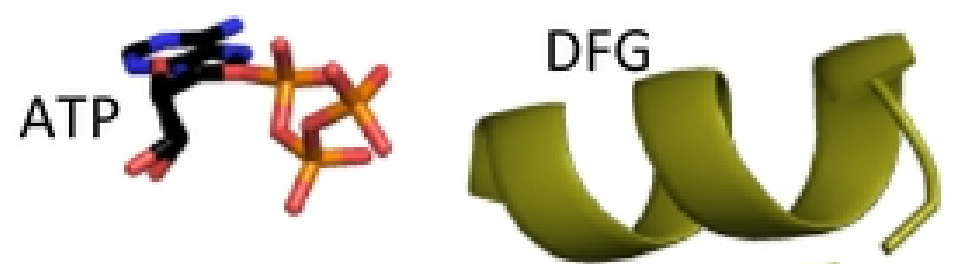

$\rightarrow$ COR-B

Full-length LRRK2

Activation Loop

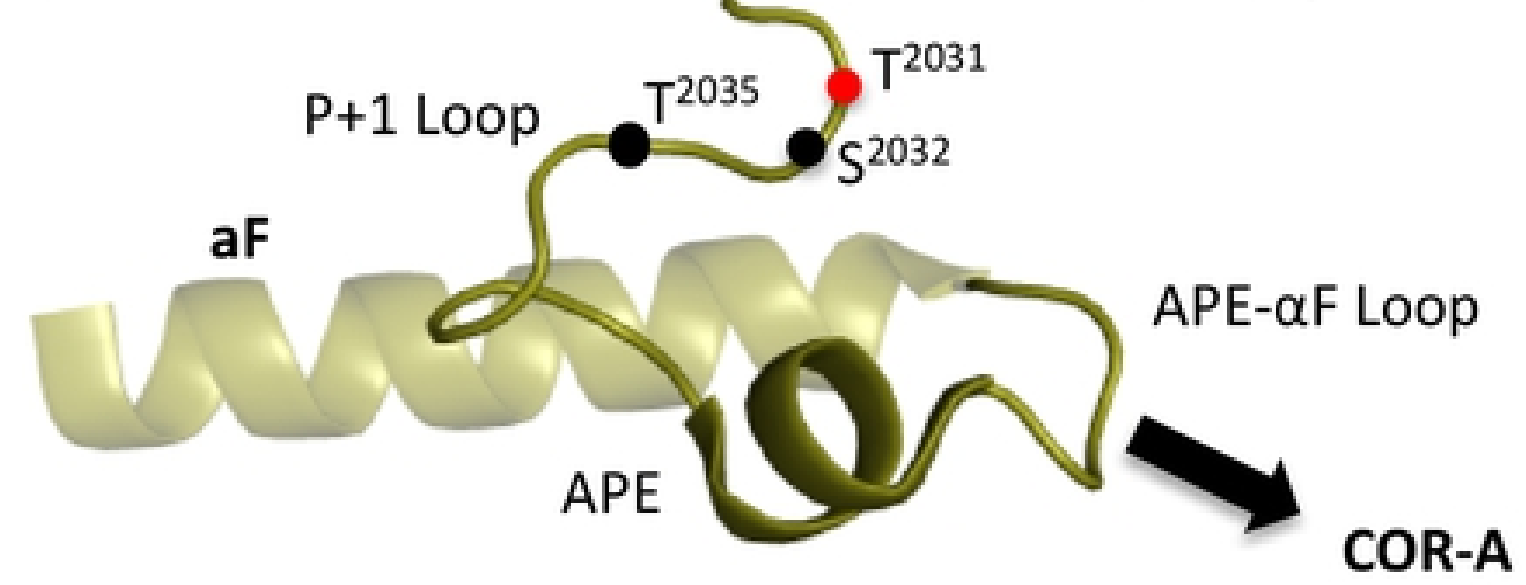

Figure 7. Comparison of Activation Segments of PKA and LRRK2. (a) The motifs that are embedded in the AS of active PKA are summarized (PDB: 1ATP). The AS begins with the DFG motif and ends with the APE motif, two of the most highly conserved motifs in the protein kinase superfamily. In between these two motifs are the A-Loop and the P+1 Loop. (b) The AS in the inactive LRRK2RCKW structure is mostly disordered. (c) The AS in the inactive full-length LRRK2 is mostly ordered, only residue 2028-2030 are missing. The A-Loop phosphate in PKA, pT197, is a red sphere and the corresponding residue in LRRK2 is also a red sphere. Additional P-sites in LRRK2 are shown as black spheres. 
a

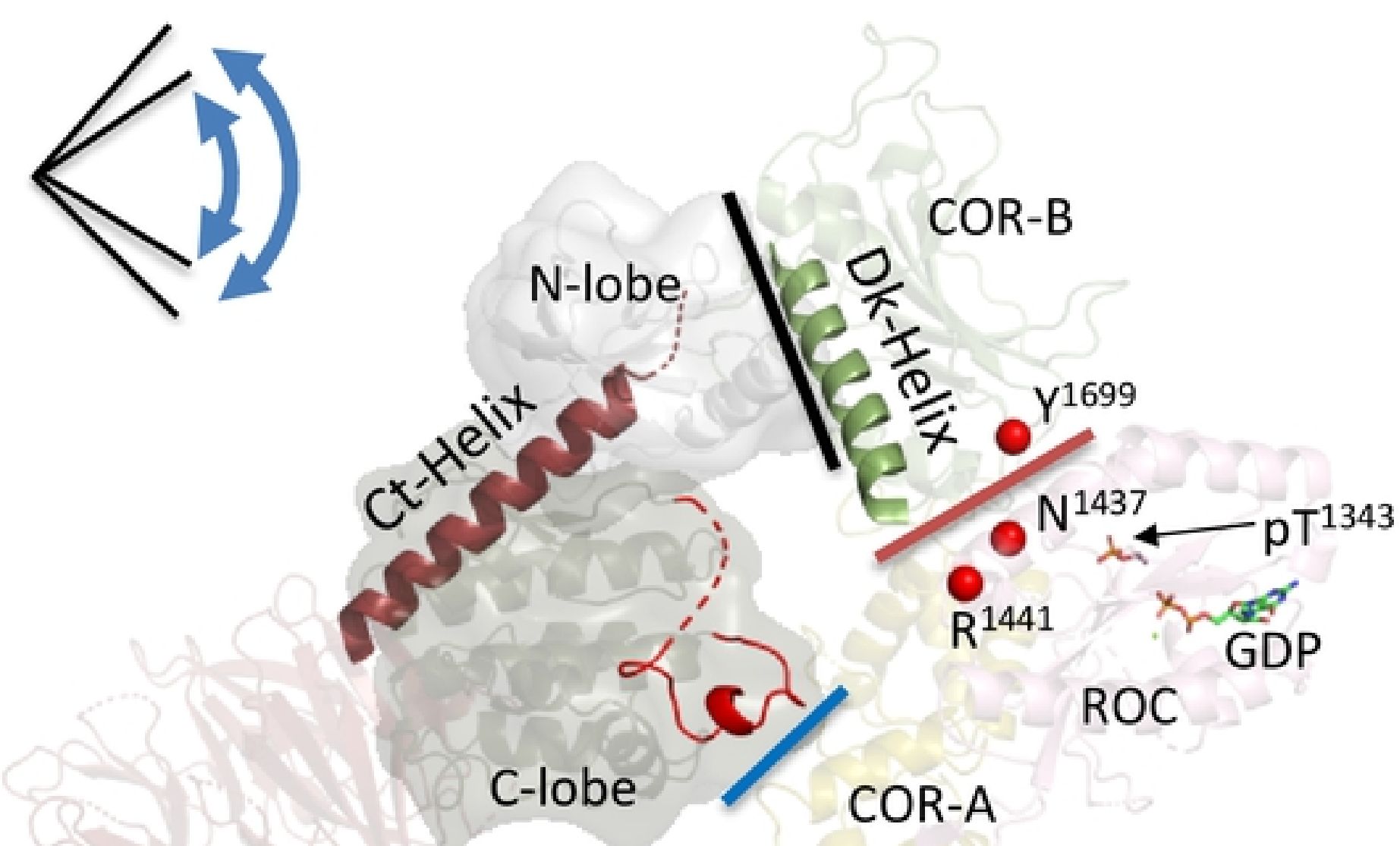

WD40

b

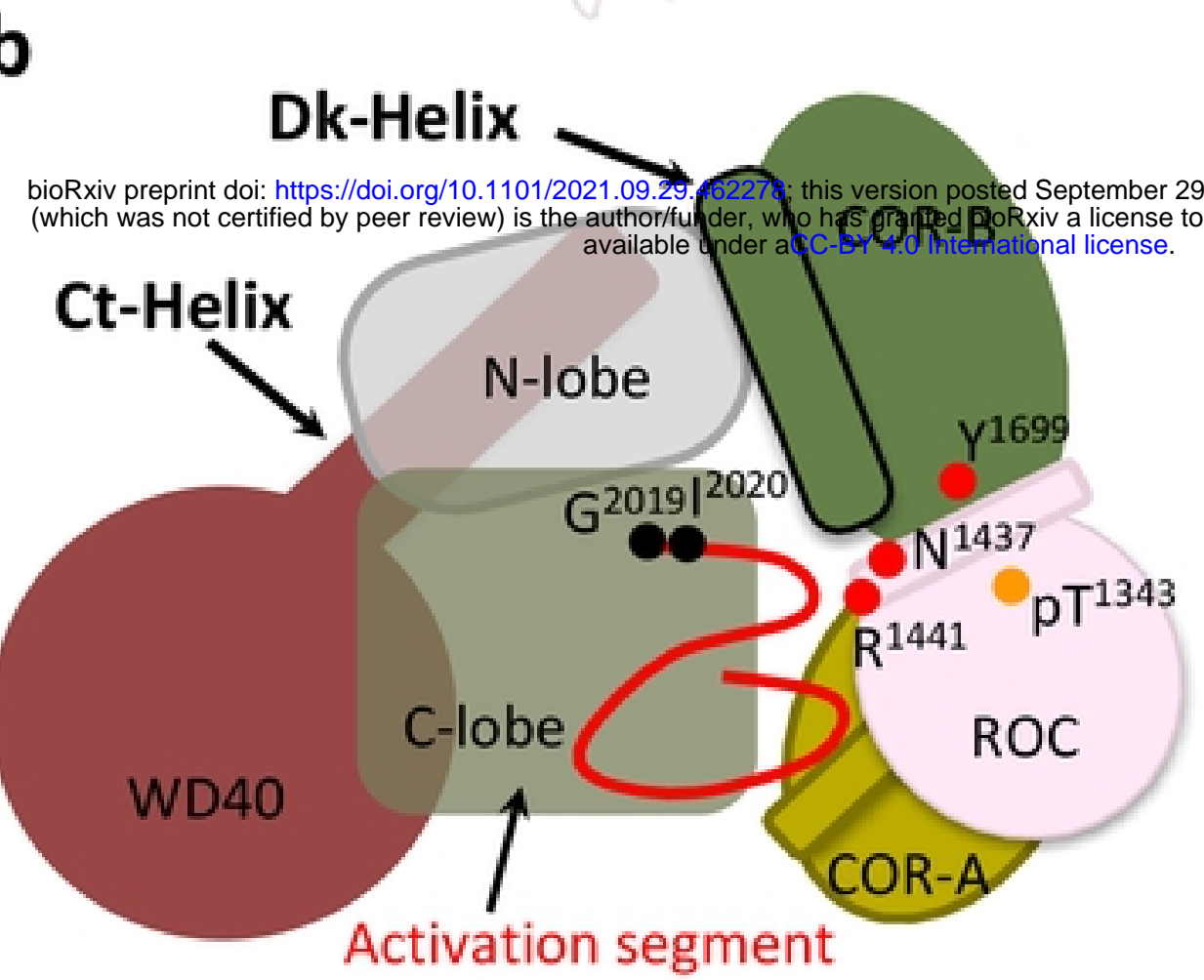

Compact

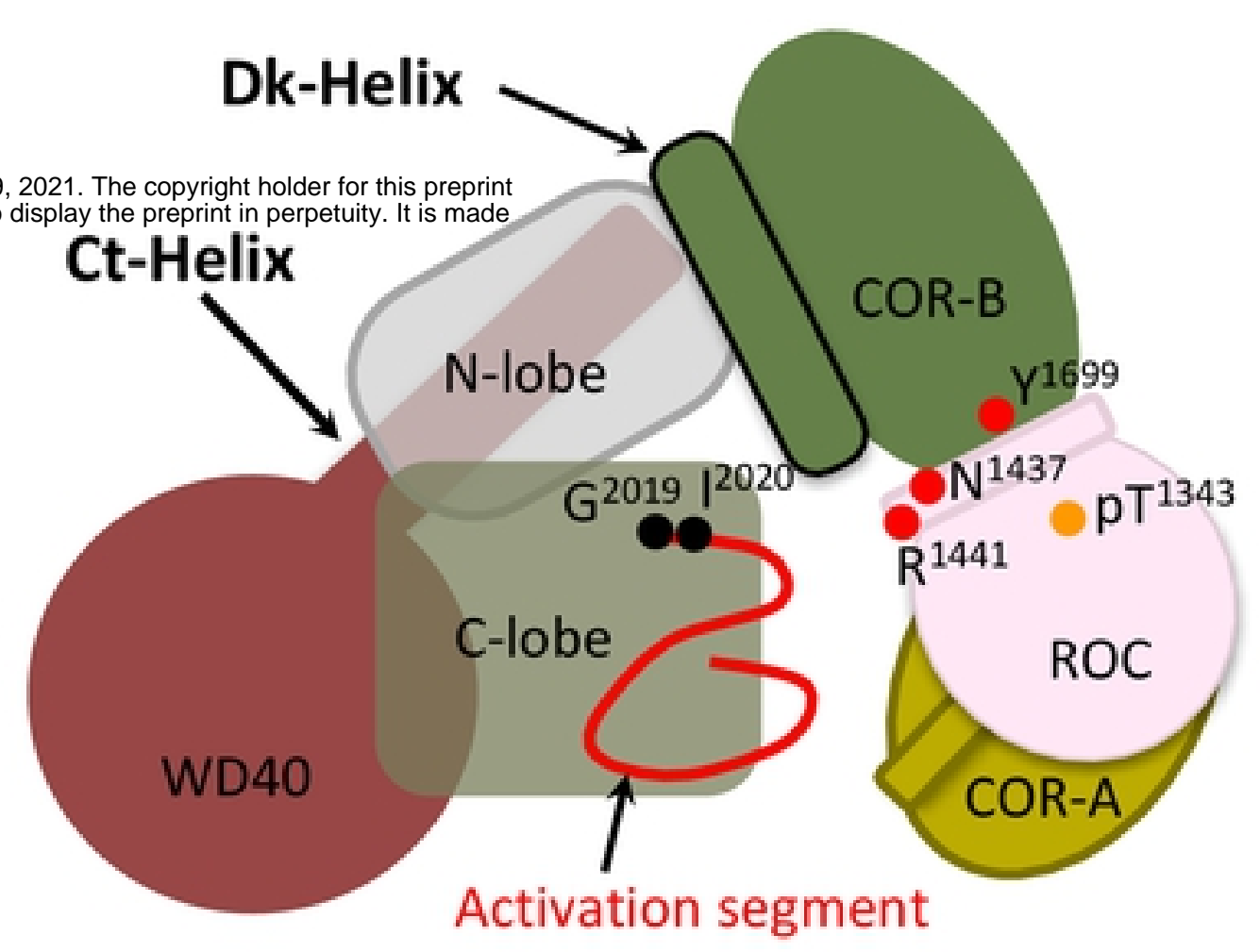

Extended

Figure 8. The interfaces in compact or extend conformation of LRRK2 $\mathbf{R C K W}_{\text {- }}$ (a) The Dk-Helix and CtHelix are highlighted on the cryo-EM structure of LRRK2RCKW. The pathogenic mutations N1437, R1441 and Y1699 are shown as red spheres. The lines show the domain: domain interfaces: COR-A domain:C-lobe (blue); ROC domain:COR-B domain (red); COR-B domain:N-lobe (black). The kinase domain toggles between open and closed conformations that lead to the compact or extended states of LRRK2RCKW. (b) Cartoon representation of the compact and extended states of LRRK2RCKW. The interaction between the COR-B domain and the N-lobe of the kinase domain, and the ROC domain as well as the COR-B domain remain intact when the COR-A domain moves away from the C-lobe of the kinase in the extended conformation. 